\title{
Buckling of Axially Compressed Cones with Imperfect Axial Length
}

\author{
F.M. Mahidan ${ }^{\mathrm{a}}$ (D), O. Ifayefunmi ${ }^{\mathrm{b} *}$ (D) \\ ${ }^{a}$ Faculty of Mechanical Engineering, Universiti Teknikal Malaysia Melaka, Malacca, Malaysia. Emails: mardhiahmahidan@gmail.com \\ ${ }^{b}$ Faculty of Mechanical and Manufacturing Engineering Technology, Universiti Teknikal Malaysia Melaka, Malacca, Malaysia. Emails: \\ olawale@utem.edu.my
}

* Corresponding author

https://doi.org/10.1590/1679-78256189

\begin{abstract}
The paper considers the effect of imperfect length on the buckling behavior of axially compressed mild steel truncated cone. Three types of initial geometric imperfections with different number of waves along the compressed edge are analyzed, and they are: (i) sinusoidal waves, (ii) triangular waves, and (iii) square waves. A validation of experimental data from previous study and further numerical calculations are provided in this paper. A good repeatability of experimental data was revealed through the test results with only $0 \%$ to $7 \%$ of error. Numerical simulations were carried out using ABAQUS FE code. It is shown that the buckling load of the cone is differently affected by the shape and amplitude of the imperfection. Apparently, the buckling loads of analyzed cones are less sensitive when imperfection shape is triangular as compared to other imperfection shapes. Furthermore, the effect of number of waves on buckling load of axially compressed cones was investigated for the above three cases. Results indicate that the influence of number of waves on the load carrying capacity of the cone is less significant.
\end{abstract}

\section{Keywords}

Buckling, collapse, imperfect axial length, mild steel cones, axial compression, contact interaction

\section{Graphical Abstract}

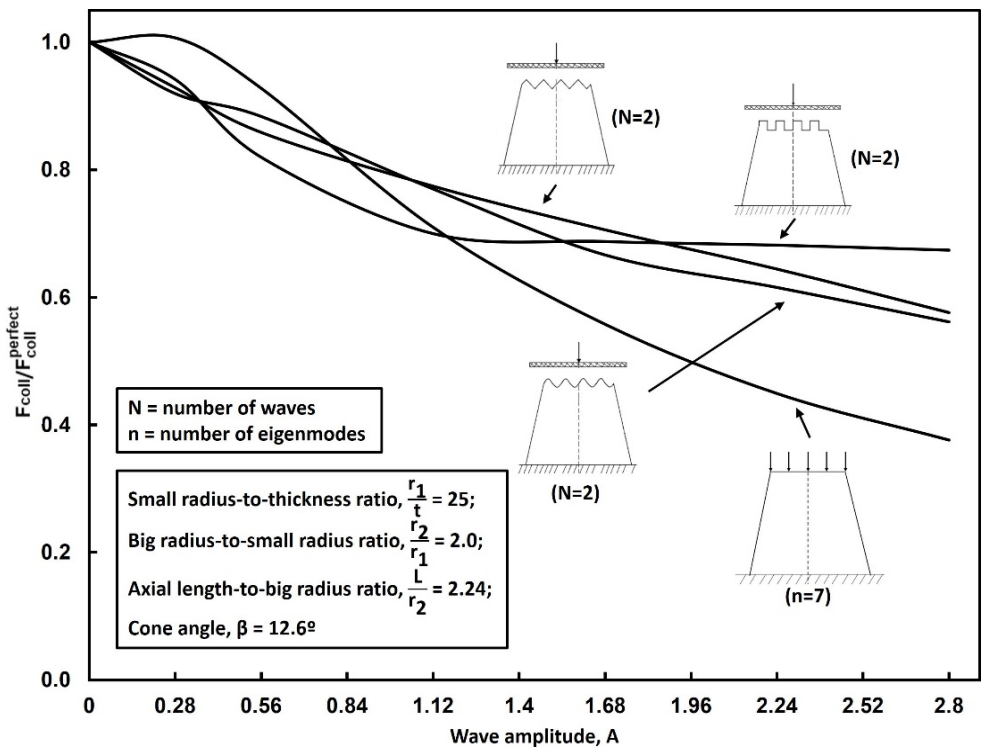




\section{INTRODUCTION}

Thin-walled conical structures find applications in major engineering structures such as aeronautical, civil engineering, offshore and pipeline structures. For instance, in aeronautical application, they are used as primary structures in launch vehicle system (such as payload adapters) where they are subjected to axial load as a result of the weight of the upper structural components and propulsive loads during launch. Whilst, in civil engineering, this structures finds application as tanks and interstage skirts. Whereas in offshore and pipeline application, they are used as (i) transition element between two cylinders of different diameter, (ii) piles for holding jackets when driven into the seabed, and (iii) legs of offshore drilling rigs. When used for such applications, the contact interaction between two neighboring shell structures becomes very important - since the safe performance of such structures are generally believed to be sensitive to imperfection such as initial geometric imperfection, imperfect length, imperfect boundary condition, crack/material discontinuity etc. (Maali, Showkati, and Mahdi Fatemi 2012). However, the degree of sensitivity of conical shell structures to imperfection is said to be strongly dependent on its geometric parameter such as the lengthto-radius ratio, the cone angle and the boundary conditions (Goldfeld, Sheinman, and Baruch 2003). One of the primary aim of any designer is to examine the different parameters that affect the shell's sensitivity, thereby improving the behavior of the structure as a whole. This technical challenge has sprung up many research on the imperfection sensitivity of conical shells structures. Review of past literatures on imperfection sensitivity of conical shells can be found in (Ifayefunmi and Błachut 2018; Ifayefunmi 2014). Generally, the most widely considered type of imperfection is the initial geometric imperfection such as eigenmode shape imperfection, axisymmetric outward bulge, localized smooth dimple and simple perturbation load. References into the influence of initial geometric imperfection on the load carrying capacity of conical shells can be found in (Błachut 2013; 2012; Ifayefunmi and Błachut 2011; 2013; Khakimova et al. 2014; Jabareen and Sheinman 2006; Castro et al. 2015; Goldfeld 2007; Ifayefunmi, Mahidan, and Maslan 2020). Moreover, few studies have been devoted to other forms of imperfections such as imperfect boundary condition (Spagnoli 2003; Chryssanthopoulos and Spagnoli 1997; Castro et al. 2014; Goldfeld and Arbocz 2004; Ifayefunmi et al. 2018), crack/material discontinuity on the structures (Ali 2013; Ifayefunmi 2017; Ifayefunmi and Ibrahim 2018).

Surprisingly, it is evident from literature survey, that there is limited information on the influence of manufacturedinduced imperfection such as uneven/imperfect length and shape on the buckling behaviour of shells structures. In fact, there is no information on the imperfection sensitivity of conical shells having uneven axial length. The only available information is on imperfect cylinder having uneven geometry, (Błachut 2010; 2015; Ifayefunmi and Zulkefli 2019; Ning and Pellegrino 2013; 2015; 2017). Błachut (2010; 2015) and Ifayefunmi and Zulkefli (2019) were devoted to influence of waves on the compressed edge of the cylinder, while, Ning and Pellegrino $(2013 ; 2015 ; 2017)$ on the other hand examine the effect of waves on the cross-sections of the cylinders (i.e., around the cylinder circumference). In (Błachut 2010), the effect of imperfect length having sinusoidal profile towards the buckling behaviour of axially compressed cylinders was investigated numerically. The sinusoidal shape was located along the compressed edge of the cylinder. Numerical results obtained in (Błachut 2010) show rapid drop of buckling strength for small axial imperfection amplitude as compared to the perfect cylinder. Błachut (2015) on the other hand presents experimental and numerical study on the imperfection sensitivity of axially compressed mild steel cylinders having variable length at one end in the form of sinusoidal wave. Eighteen steel cylindrical shells were machined in batches of three using CNC machining process. Cylinders were designed to have wave numbers, $\mathrm{N}=3,6$ and 9, and five different imperfection amplitude, $2 \mathrm{~A}=0.02 \mathrm{~mm}, 0.04 \mathrm{~mm}, 0.1 \mathrm{~mm}, 0.2 \mathrm{~mm}$ and $0.4 \mathrm{~mm}$. The specimens were assumed to have a nominal thickness of $0.4 \mathrm{~mm}$. Again, rapid drop of the load carrying capacity of the cylinder for small imperfection amplitude was reported. Furthermore, the results of cylinders' imperfect length were benchmarked with eigenmode shape of the same imperfection amplitude. It was found that buckling strength of axially compressed cylindrical shells is severely reduced in the case of cylinders which have imperfect length and that the load bearing capacity of 'axially imperfect' cylinders can be up to five times smaller than that of eigenmode imperfection. In (Ifayefunmi and Zulkefli 2019), the buckling behaviour of axially compressed mild steel cylindrical shells with non-uniform axial length having sinusoidal and triangular waves were conducted through experimental tests and numerical computation using ABAQUS FE code. Nineteen steel cylinders with imperfect length were manufactured from $1 \mathrm{~mm}$ steel plate and welded using MIG welding techniques. The radius-to-thickness ration of the cylinder was assumed to be 50 and the number of waves, N, was set to 4, 6, 8, 10 and 12. The axial imperfection amplitude, A, of the cylinders was varied from $0-2.8$. Although, for experimental purposes, only cylinders with imperfection amplitude, $A=0.28$ was considered. Results confirms that the introduction of non-uniform axial length on the cylindrical structures are proven to decrease the collapse load of the perfect cylinders. Also, it was reported that axially compressed cylinders having triangular waves at the compressed edge are less sensitive to imperfection when compared to cylinders having sinusoidal waves. Moreover, in (Ning and Pellegrino 2013; 2015; 2017), the imperfection sensitivity of composite cylinders having wavy cross section subjected to axial compression were discussed. An alternative approach to improve the imperfection sensitivity of cylindrical shells has been proposed in (Ning and Pellegrino 2013) which was based on symmetry-breaking wavy cylindrical shells. This design was formulated by NURBS interpolation on control points whose positions are optimized by evolutionary algorithms. In (Ning and Pellegrino 2013), two perfect circular cylindrical shells and four imperfect cylindrical shell with wavy cross-section were clamped at both ends and subjected to axial compression. The cylindrical shells have an average thickness of 0.170 $\mathrm{mm}$ and average imperfection amplitude ranging from $0.928 \mathrm{~mm}$ to $1.128 \mathrm{~mm}$. The shells undergo curing process in order to form waves on imperfect cylindrical models. The results from experimental were validated using numerical computations 
on ABAQUS software. The optimization design reveals that composite cylindrical shells having wavy cross section to practically have imperfection-insensitive behaviour having knockdown factors about 0.995. Based on the mass efficiency studies, the said structural design was more efficient than even a perfect circular cylindrical shell and most stiffened cylindrical shells. In 2015, a numerical analysis was conducted in (Ning and Pellegrino 2015) using the previously stated design criteria of composite cylindrical shells with wavy cross-section in (Ning and Pellegrino 2013) by adding comparison to alternative shell designs (e.g. sinusoidal corrugated shells, aster shell) and computing the mass efficiency of the suggested model. This study confirmed that cylindrical shells manufactured with this design approach can achieve higher critical stresses and knockdown factors value than any other known cylindrical shells while also being practically insensitive to geometric imperfections. In (Ning and Pellegrino 2017), tests data alongside with their numerical simulations on axially compressed cylinder with wavy cross section were presented. Three specimens of different imperfection amplitude ranging from $0.374 \mathrm{~mm}$ to $0.536 \mathrm{~mm}$ with nominal shell thickness of $0.180 \mathrm{~mm}$ were tested under axial compression. Numerical computations using ABAQUS were carried out to confirm the results and it was proven that the wavy shells are not sensitive towards imperfection. In general, axially compressed cylindrical shells having wave imperfection at the compressed edge are said to be sensitive to imperfection, while cylindrical shells having wave imperfection at the cylinder's cross-sections are seen to be imperfection insensitive. In practice, thin-shell with imperfection in the form of imperfect axial length on the compressed edge appears to be more realistic since this cannot be totally avoided during the manufacturing process. Hence, the question on the role of imperfect length on the buckling behaviour of conical shells is a valid one. The current paper examines the imperfection sensitivity of truncated mild steel cone having uneven length subjected to axial compression. The effect of (i) increasing the axial imperfection amplitude, A, (ii) increasing the number of waves, and (iii) wave profiles (sinusoidal, triangular and square waves) on the buckling load of axially compressed conical shell is presented. Also, the effect of contact interaction problem between the cones and the rigid plate is discussed. This is purely numerical work based on the ABAQUS FE code (Hibbit, Karlsson, and Sorensen 2006) and it complements the experimental programme, results of which are given in (Ifayefunmi, Mahidan, and Wang 2019; Mahidan and Ifayefunmi 2020).

\section{BACKGROUND - EXPERIMENTAL DATA}

A brief summary of the experimental programme is presented in this section for completeness purposes. A total of thirty-two laboratory scale conical shells were manufactured from $1 \mathrm{~mm}$ mild steel plate and tested under axial compression were presented in (Ifayefunmi, Mahidan and Wang 2019, Mahidan and Ifayefunmi 2020). The first two cones were assumed to be perfect with no wave on the compressed edge, while ten conical shells each were allocated for every wave profiles (i.e., sinusoidal wave, triangular wave, and square wave). The geometric parameter of cone was taken as: big radius-to-small radius ratio, $r_{2} / r_{1}=2.0$; small radius-to-thickness ratio, $r_{1} / t=25$; axial length-to-big radius ratio, $L / r_{2}=2.24$; nominal wall thickness, $t=1 \mathrm{~mm}$, cone angle, $\beta=12.6^{\circ}$, and axial imperfection amplitude, $A=0.28 \mathrm{~mm}$ as shown in Figure 1. The material properties of the mild steel plate was obtained from experiment, they are: Young's Modulus $\mathrm{E}=168.791 \mathrm{GPa}$, yield stress, $\sigma_{\mathrm{yp}}=229.774 \mathrm{MPa}$. While, Poisson ratio, $\mathrm{v}=0.3$ was taken from material data sheet. This material data will be used later for numerical calculations. Further details on manufacturing, material and axial testing process of cones can be found in (Ifayefunmi, Mahidan and Wang 2019). Manufacture-induced imperfections were taken into account before the axial testing process was carried out.

Several measurements (i.e., wall thickness, diameter, axial length and slant length) of all the conical models were taken. Firstly, micrometer screw gauge was used to measure the wall thickness of the cones at eleven (11) equal points along the axial meridian. This was then repeated along the circumference of the cone at $36^{\circ}$ apart, resulting in $11 \times 10=110$ measuring points. The minimum thickness $t_{\min }$, maximum thickness $t_{\max }$, average thickness $t_{a v e}$ and the standard deviation $t_{\text {std }}$ for all the manufactured cones can be found in Table 1. Secondly, a digital Vernier caliper was used to measure the inner and outer diameters of the specimens. Five equally spaced diameters were measured at the top and bottom ends respectively. The average measured mid-surface diameter for all specimens are also given in Table 1. Thirdly, the axial length and slant length of the cones at eleven equal point were measured using a digital Vernier caliper. The measured average axial length and slant length are in column 6 and 7 respectively in Table 1 . These shape measurements were assumed to represent the most obvious characteristic of the specimens.

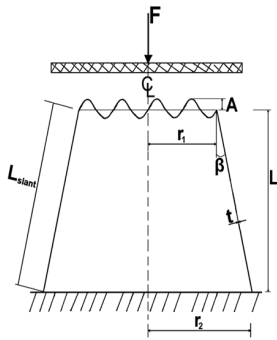

(a)

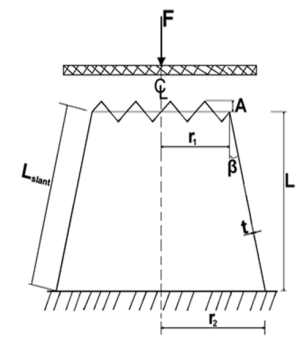

(b)

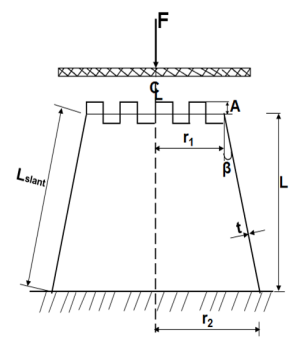

(c)

Figure 1. Geometry of the analyzed cone having non-uniform length with number of waves, $N=4$, in the form of sinusoidal wave (Figure 1a), triangular waves (Figure 1b), and square waves (Figure 1c). 
Figure 2 depicts the plot of experimental buckling load against the number of waves for all the tested cones. The plot of experimental buckling load for each conical pairs versus the number of waves corresponding to different imperfection profile are presented in Figures 3-5 for cones with sinusoidal, triangular and square waves, respectively. The magnitude of the collapse load for all the tested cones is given in Table 2. From Figures 3-5, it can be observed that nominally identical conical shell failed with similar collapse load. The percentage error in collapse load within each pair were: $2 \%$ (model 1 vs 2 ), $4 \%$ (model 3 vs 4 ), $2 \%$ (model 5 vs 6 ), $2 \%$ (model 7 vs 8 ), $3 \%$ (model 9 vs 10 ), $3 \%$ (model 11 vs 12 ), $4 \%$ (model 13 vs 14), $2 \%$ (model 15 vs 16 ), $1 \%$ (model 17 vs 18 ), $2 \%$ (model 19 vs 20), 3\% (model 21 vs 22 ), 0\% (model 23 vs 24 ), $0 \%$ (model 25 vs 26 ), $1 \%$ (model 27 vs 28 ), $1 \%$ (model 29 vs 30 ) and $1 \%$ (model 31 vs 32 ). From the results, comparison of experimental results for each pairs are seen to be very good especially for imperfect cones with square waves as shown in Figure 5. Hence, confirming repeatability of experimental data. The largest error of $7 \%$ was found in conical shells with sinusoidal waves, $\mathrm{N}=10$ - models 9 and 10 (see Figure 3), while the smallest error of $0 \%$ is observed twice in conical shells with square waves, $\mathrm{N}=4$ - models 13 and 14 , and $\mathrm{N}=6-$ models 15 and 16 (see Figure 5 ).

Table 1. Measurements of all tested conical shells.

\begin{tabular}{|c|c|c|c|c|c|c|c|c|c|c|c|}
\hline \multirow{2}{*}{ Model } & \multirow{2}{*}{$\mathbf{N}$} & \multirow{2}{*}{ A } & $2 r_{1}$ & $2 r_{2}$ & $\mathbf{L}$ & $\mathrm{L}_{\text {slant }}$ & $t_{\min }$ & $t_{\max }$ & $t_{\text {ave }}$ & \multirow{2}{*}{$t_{\text {std }}$} & \multirow{2}{*}{ Wave shape } \\
\hline & & & \multicolumn{4}{|c|}{$(\mathrm{mm})$} & \multicolumn{3}{|c|}{$(\mathrm{mm})$} & & \\
\hline 1 & 0 & 0 & 49.71 & 99.02 & 112.99 & 114.77 & 0.95 & 0.96 & 0.96 & 0.0050 & Perfect \\
\hline 2 & 0 & 0 & 49.53 & 98.78 & 112.71 & 114.73 & 0.95 & 0.96 & 0.96 & 0.0050 & Perfect \\
\hline 3 & 4 & 0.28 & 49.05 & 97.67 & 112.41 & 114.45 & 0.95 & 0.99 & 0.96 & 0.0081 & Sinusoidal \\
\hline 4 & 4 & 0.28 & 49.68 & 99.21 & 112.50 & 114.65 & 0.95 & 0.97 & 0.96 & 0.0085 & Sinusoidal \\
\hline 5 & 6 & 0.28 & 49.62 & 98.69 & 112.69 & 114.76 & 0.95 & 0.97 & 0.96 & 0.0057 & Sinusoidal \\
\hline 6 & 6 & 0.28 & 50.52 & 99.19 & 112.75 & 114.78 & 0.95 & 0.97 & 0.96 & 0.0060 & Sinusoidal \\
\hline 7 & 8 & 0.28 & 49.72 & 99.05 & 112.47 & 114.58 & 0.95 & 0.97 & 0.96 & 0.0089 & Sinusoidal \\
\hline 8 & 8 & 0.28 & 49.52 & 98.83 & 112.59 & 114.59 & 0.95 & 0.99 & 0.96 & 0.0095 & Sinusoidal \\
\hline 9 & 10 & 0.28 & 49.69 & 98.86 & 112.59 & 114.76 & 0.95 & 0.97 & 0.96 & 0.0060 & Sinusoidal \\
\hline 10 & 10 & 0.28 & 51.05 & 98.44 & 112.85 & 114.76 & 0.95 & 0.98 & 0.95 & 0.0056 & Sinusoidal \\
\hline 11 & 12 & 0.28 & 50.01 & 98.88 & 112.82 & 114.74 & 0.95 & 1.01 & 0.96 & 0.0082 & Sinusoidal \\
\hline 12 & 12 & 0.28 & 49.39 & 98.76 & 112.80 & 114.76 & 0.95 & 1.08 & 0.96 & 0.0143 & Sinusoidal \\
\hline 13 & 4 & 0.28 & 51.16 & 99.80 & 111.89 & 114.55 & 0.95 & 0.97 & 0.96 & 0.0057 & Triangular \\
\hline 14 & 4 & 0.28 & 50.60 & 99.52 & 111.81 & 114.45 & 0.95 & 0.97 & 0.96 & 0.0054 & Triangular \\
\hline 15 & 6 & 0.28 & 50.58 & 99.81 & 111.85 & 114.40 & 0.95 & 0.97 & 0.96 & 0.0047 & Triangular \\
\hline 16 & 6 & 0.28 & 50.82 & 100.67 & 111.86 & 114.48 & 0.95 & 0.97 & 0.96 & 0.0053 & Triangular \\
\hline 17 & 8 & 0.28 & 50.03 & 99.11 & 111.78 & 114.42 & 0.95 & 0.97 & 0.96 & 0.0048 & Triangular \\
\hline 18 & 8 & 0.28 & 50.55 & 99.48 & 111.76 & 114.35 & 0.95 & 0.97 & 0.96 & 0.0052 & Triangular \\
\hline 19 & 10 & 0.28 & 50.15 & 99.62 & 111.68 & 114.49 & 0.95 & 0.97 & 0.96 & 0.0040 & Triangular \\
\hline 20 & 10 & 0.28 & 50.37 & 100.04 & 112.00 & 114.29 & 0.95 & 0.97 & 0.96 & 0.0055 & Triangular \\
\hline 21 & 12 & 0.28 & 50.26 & 99.45 & 111.48 & 113.99 & 0.95 & 0.97 & 0.96 & 0.0043 & Triangular \\
\hline 22 & 12 & 0.28 & 50.28 & 99.26 & 111.68 & 114.31 & 0.95 & 0.97 & 0.96 & 0.0052 & Triangular \\
\hline 23 & 4 & 0.28 & 50.19 & 99.25 & 111.64 & 113.92 & 0.95 & 0.97 & 0.96 & 0.0051 & Square \\
\hline 24 & 4 & 0.28 & 50.11 & 98.99 & 111.82 & 114.29 & 0.95 & 0.97 & 0.96 & 0.0049 & Square \\
\hline 25 & 6 & 0.28 & 50.90 & 99.62 & 111.77 & 114.36 & 0.95 & 0.97 & 0.96 & 0.0053 & Square \\
\hline 26 & 6 & 0.28 & 50.42 & 99.65 & 111.29 & 114.37 & 0.95 & 0.97 & 0.96 & 0.0049 & Square \\
\hline 27 & 8 & 0.28 & 50.23 & 99.40 & 111.79 & 114.53 & 0.95 & 0.97 & 0.96 & 0.0051 & Square \\
\hline 28 & 8 & 0.28 & 49.99 & 99.52 & 112.05 & 114.43 & 0.95 & 0.97 & 0.96 & 0.0054 & Square \\
\hline 29 & 10 & 0.28 & 49.93 & 99.21 & 111.29 & 114.58 & 0.95 & 0.97 & 0.96 & 0.0045 & Square \\
\hline 30 & 10 & 0.28 & 50.57 & 99.90 & 112.07 & 114.50 & 0.95 & 0.97 & 0.96 & 0.0039 & Square \\
\hline 31 & 12 & 0.28 & 50.27 & 99.42 & 112.09 & 114.49 & 0.95 & 0.97 & 0.96 & 0.0041 & Square \\
\hline 32 & 12 & 0.28 & 50.28 & 99.68 & 111.94 & 114.54 & 0.95 & 0.97 & 0.96 & 0.0049 & Square \\
\hline
\end{tabular}




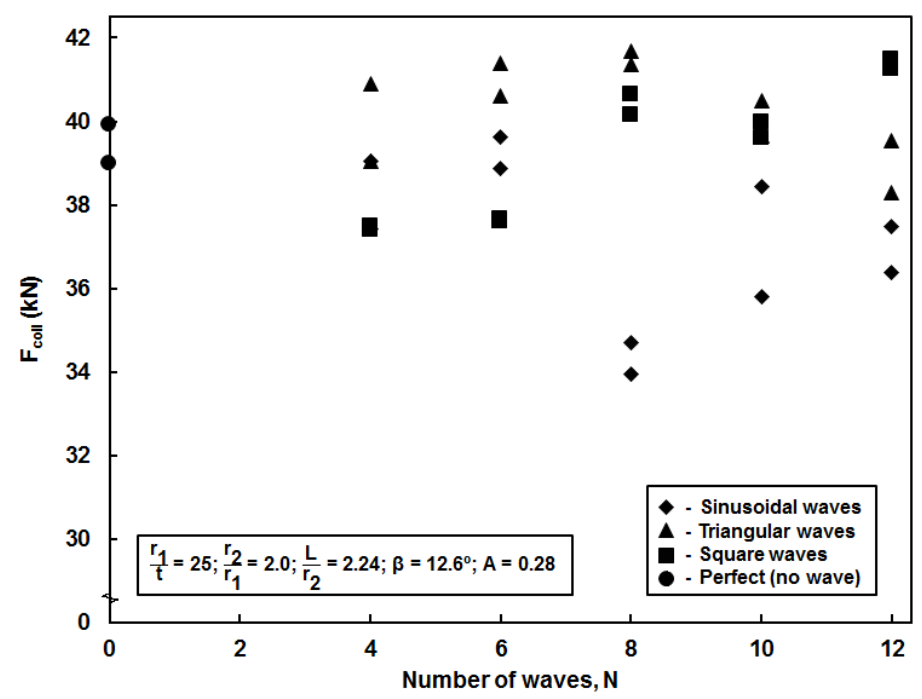

Figure 2. Plot of experimental collapse load of perfect and imperfect cones having different wave profiles and different number of wave, $\mathrm{N}$.

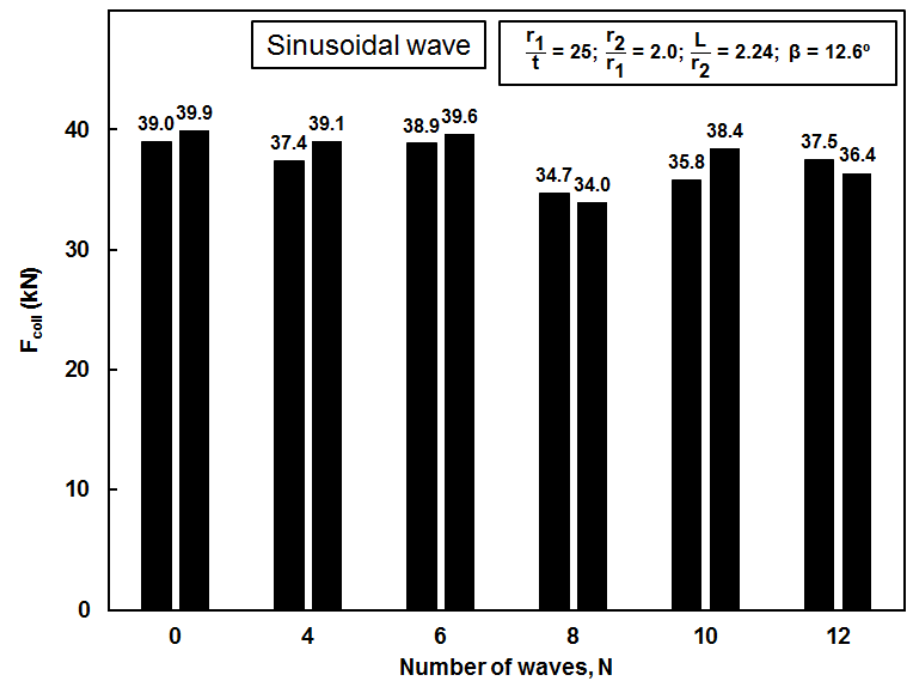

Figure 3. Plot of experimental collapse load of perfect and imperfect cones having sinusoidal wave profiles with different number of wave, $\mathrm{N}$.

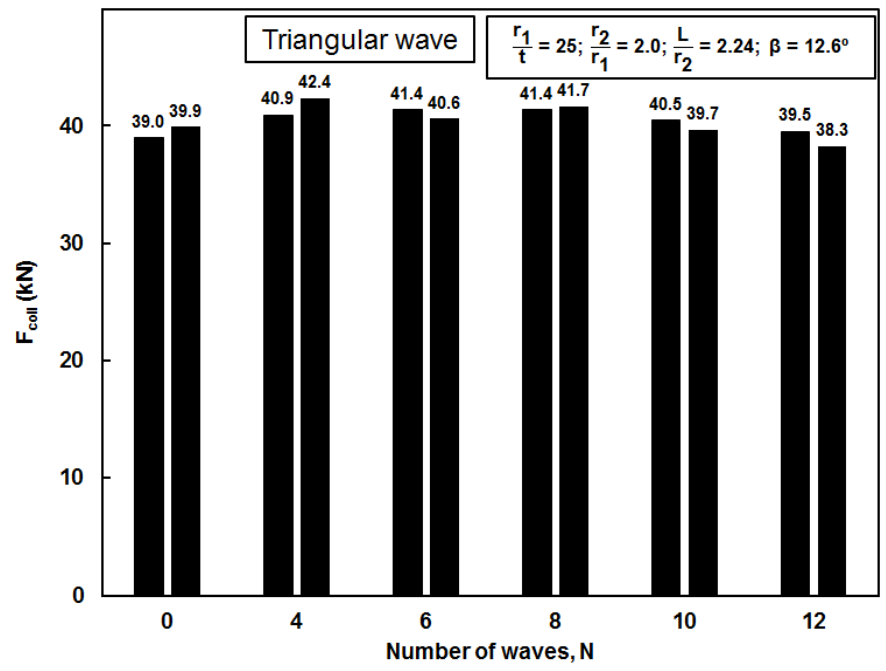

Figure 4. Plot of experimental collapse load of perfect and imperfect cones having triangular wave profiles with different number of wave, $\mathrm{N}$. 


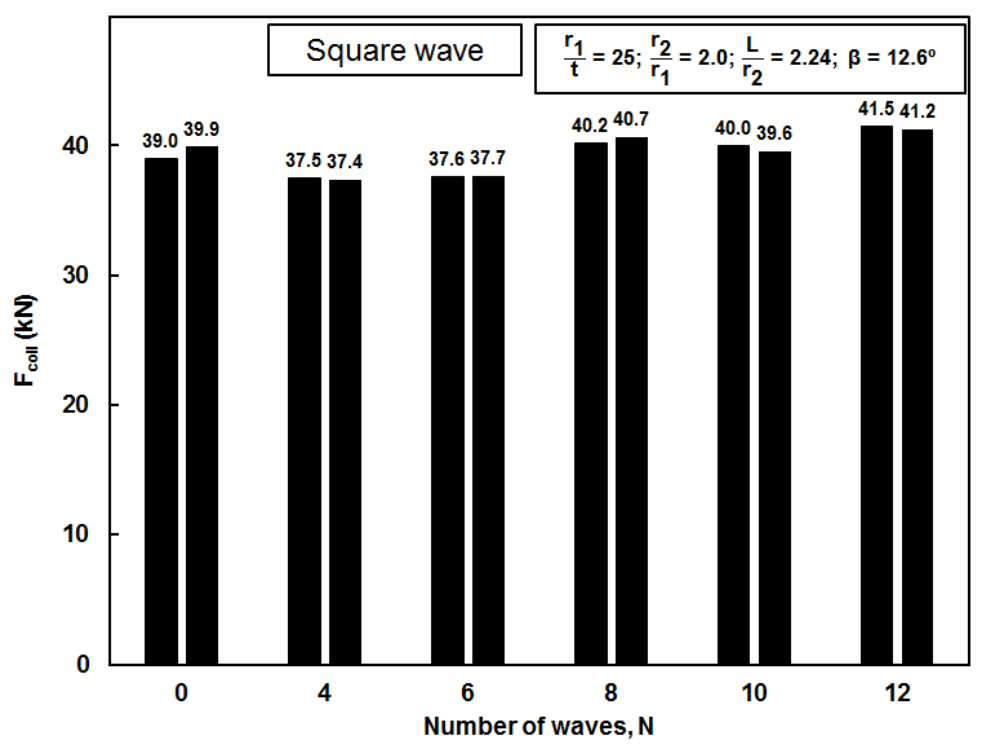

Figure 5. Plot of experimental collapse load of perfect and imperfect cones having square wave profiles and number of wave, $\mathrm{N}$.

Table 2. Comparison of experimental and numerical buckling load of imperfect cones with different wave number and wave shape.

\begin{tabular}{|c|c|c|c|c|c|}
\hline \multirow{2}{*}{ Model } & \multirow{2}{*}{$\mathbf{N}$} & \multicolumn{2}{|c|}{ Buckling load of cone (kN) } & \multirow{2}{*}{$\%$ difference } & \multirow{2}{*}{ Wave shape } \\
\hline & & Exptl & ABAQUS & & \\
\hline 1 & 0 & 38.99 & 36.14 & 7.3 & Perfect \\
\hline 2 & 0 & 39.91 & 36.11 & 9.5 & Perfect \\
\hline 3 & 4 & 37.41 & 34.54 & 7.7 & Sinusoidal \\
\hline 4 & 4 & 39.06 & 34.38 & 12 & Sinusoidal \\
\hline 5 & 6 & 38.86 & 34.07 & 12.3 & Sinusoidal \\
\hline 6 & 6 & 39.64 & 34.1 & 14 & Sinusoidal \\
\hline 7 & 8 & 34.71 & 34.45 & 0.7 & Sinusoidal \\
\hline 8 & 8 & 33.96 & 34.56 & 1.8 & Sinusoidal \\
\hline 9 & 10 & 35.79 & 34.03 & 4.9 & Sinusoidal \\
\hline 10 & 10 & 38.43 & 33.97 & 11.6 & Sinusoidal \\
\hline 11 & 12 & 37.47 & 34.35 & 8.3 & Sinusoidal \\
\hline 12 & 12 & 36.4 & 34.42 & 5.4 & Sinusoidal \\
\hline 13 & 4 & 40.91 & 34.71 & 15.1 & Triangular \\
\hline 14 & 4 & 42.35 & 34.69 & 18.1 & Triangular \\
\hline 15 & 6 & 41.39 & 34.35 & 17 & Triangular \\
\hline 16 & 6 & 40.61 & 34.39 & 15.3 & Triangular \\
\hline 17 & 8 & 41.36 & 34.5 & 16.6 & Triangular \\
\hline 18 & 8 & 41.67 & 34.48 & 17.3 & Triangular \\
\hline 19 & 10 & 40.5 & 34.53 & 14.7 & Triangular \\
\hline 20 & 10 & 39.65 & 34.53 & 12.9 & Triangular \\
\hline 21 & 12 & 39.53 & 34.49 & 12.8 & Triangular \\
\hline 22 & 12 & 38.3 & 34.47 & 10 & Triangular \\
\hline 23 & 4 & 37.49 & 34.11 & 9 & Square \\
\hline 24 & 4 & 37.39 & 34.09 & 8.8 & Square \\
\hline 25 & 6 & 37.61 & 33.69 & 10.4 & Square \\
\hline 26 & 6 & 37.65 & 33.62 & 10.7 & Square \\
\hline 27 & 8 & 40.16 & 34.78 & 13.4 & Square \\
\hline 28 & 8 & 40.65 & 34.77 & 14.5 & Square \\
\hline 29 & 10 & 39.96 & 33.22 & 16.9 & Square \\
\hline 30 & 10 & 39.58 & 33.23 & 16.1 & Square \\
\hline 31 & 12 & 41.48 & 33.73 & 18.7 & Square \\
\hline 32 & 12 & 41.24 & 33.71 & 18.3 & Square \\
\hline
\end{tabular}


Figure 6 depicts the comparison of average experimental collapse load for each wave's profile. Here, the collapse force for imperfect cones were normalized by the collapse force of perfect cones (i.e., $F_{\text {coll }} / F_{\text {coll }}^{\text {perfect }}$ ). From Figure 6 , it is evident that triangular waves are the least sensitive to imperfection as compared to other wave profiles. This observation is the same with that of imperfect cylinders reported in (Ifayefunmi and Zulkefli 2019). Surprisingly, two distinct regions are seen for cones having sinusoidal and square waves, (i.e. region ' $A$ ' and region ' $B$ '). As can be seen from Figure 6 , at region ' $A$ ', where the number of waves, $N$ is small, cones having square waves show more sensitivity towards imperfection. However, at region ' $\mathrm{B}$ ', as the number of waves increases, cones having sinusoidal waves have a lower load carrying capacity. Generally, it can be seen that for axially compressed conical models with small amplitude of axial imperfection $(A=0.28 \mathrm{~mm})$, there is a significant reduction in the load carrying capacity of the shells. This behavior is seen to be similar to what has been reported in (Błachut 2015; Ifayefunmi and Zulkefli 2019) for imperfect cylinder with sinusoidal waves. This is quite significant from the practical point of view, because the manufactured-induced imperfection amplitude seen in real life situations are very small. In addition, it is apparent that the number of waves producing the largest drop in buckling strength for cones with different wave shape is somewhat different. As an example for cones with sinusoidal waves, largest drop of $13 \%$ was observed at $\mathrm{N}=8$, while $1 \%$ reduction was observed for cones with triangular waves having $\mathrm{N}=12$ and $5 \%$ reduction for cones with square waves with $\mathrm{N}=4$. Although, this may not be entirely true when you considered $\mathrm{N}=2$.

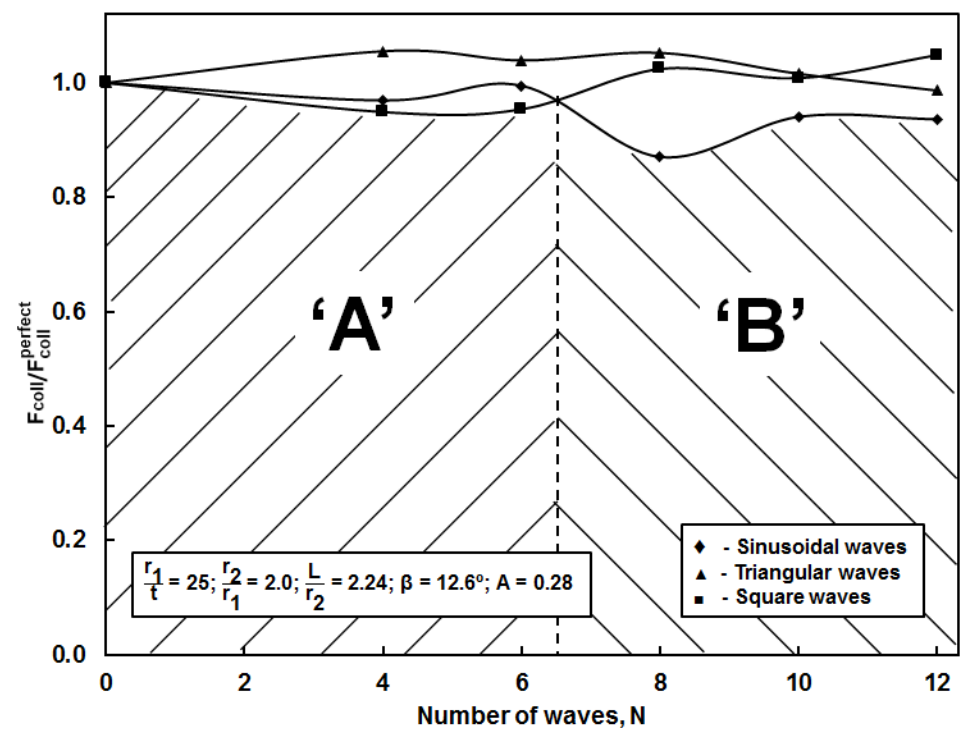

Figure 6. Plot of average experimental collapse load of perfect and imperfect cones having different wave profiles and number of wave, $\mathrm{N}$.

\section{NUMERICAL MODELLING}

Numerical validations were carried out on imperfect cones with non-uniform axial length subjected to axial compression to validate the experimental results from previous study (Ifayefunmi, Mahidan and Wang 2019; Mahidan and Ifayefunmi 2020). As depicted in Figure 1, cone is assumed to be fixed at the big radius ends, while the same condition was employed at the top ends except movement in the axial direction. Three types of imperfections profile with different number of waves along the compressed edge (i.e., the small radius end of the cone) were considered, they are: (i) sinusoidal waves (Figure 1a), (ii) triangular waves (Figure 1b), and (iii) square waves (Figure 1c). The choice of introducing uneven length at the small radius end of the cone can be attributed to the fact that for conical shells the spread of plastic strain is concentrated within the small radius end of the cone (Błachut and Ifayefunmi 2010a; Błachut, Ifayefunmi, and Corfa 2011). Conical specimens were modelled as deformable body using four noded shell elements with six degree of freedom (S4R). The numerical predictions employed the use of measured specimen data i.e., average thickness and average mid-surface diameter (Table 1). The material is modelled as elastic-perfectly plastic with the following material data: Young's Modulus $\mathrm{E}=168.791 \mathrm{GPa}$, yield stress, $\sigma_{\mathrm{yp}}=229.774 \mathrm{MPa}$ and Poisson ratio, $v=0.3$ (data taken from material data sheet). It is worth mentioning that the material data were obtained from uni-axial test as presented in (Ifayefunmi, Mahidan and Wang 2019). Axial load was applied to the small radius end of the cone through a horizontal and rigid plate moving downward as shown in Figure 1. To mimic the axial loading application during experimental testing, a reference node was defined at the center of the rigid plate. During the computation, surface-tosurface contact interaction between the rigid plate and the cone using master-slave algorithm was employed. In the 
master-slave algorithm, the rigid plate is referred to as the master, while the set of all nodes at the top edge of the cone is referred to as the slave. Then, non-linear static analysis was carried out on the conical model using modified RIKS algorithm. Mesh convergence study was carried out on perfect cone and the results is presented in Table 3 . From the study, it shows that 1872 elements were sufficient in the numerical modelling of the conical models.

Table 3. Mesh convergence studies for axially compressed perfect cone.

\begin{tabular}{cccccc}
\hline Element size & $\mathbf{2 6 0}$ & $\mathbf{5 6 0}$ & $\mathbf{9 8 8}$ & $\mathbf{1 8 7 2}$ & $\mathbf{9 0 0 6}$ \\
\hline Fcoll, (kN) & 40.05 & 38.88 & 38.14 & 37.85 & 37.82 \\
\hline
\end{tabular}

\section{RESULTS AND DISCUSSION}

\subsection{Validation of experimental data}

First, numerical computations using ABAQUS FE code was presented for the purpose of validating the experimental data presented in (Ifayefunmi, Mahidan and Wang 2019; Mahidan and Ifayefunmi 2020). The simulations employed the use of specimen measured data (i.e., average thickness and average mid-surface - as shown in Table 1). The ensuing results from the numerical computation of conical models are given in column 4 of Table 2 with their corresponding experimental buckling load. It is found from Table 2 that different wave profiles will affect the collapse load of mild steel conical shells differently. For example, at $\mathrm{N}=4$ and 6 , cones with square was produce the lowest buckling load, and as the wave number increases to $\mathrm{N}=8$, there is transition where cones with sinusoidal waves becomes the lowest. This confirm the experimental behavior depicted in Figure 6. Again, the smallest drop of buckling strength produced by axially compressed conical shells with triangular waves as compared to other waves profiles has been confirmed numerically. Comparisons between average experimental and numerical collapse load against number of waves for cones with sinusoidal waves is depicted in Figure 7. It can be seen that there is a good agreement in the comparison of collapse load of conical shells from experimental and numerical simulation. The ratio of experimental load to numerical collapse load ranges from $1 \%$ to $14 \%$ for all tested cones. The experimental results overestimates all of the numerical prediction, except for conical shell model 8 . It is hope that the use of variable geometry and variable thickness (see Table 1 ) in the numerical calculation will provide a better agreement.

Identical plots to Figure 7 for conical shells having triangular waves and square waves are presented in Figures 8 and 9, respectively. Although, there is a better comparison between experimental data for cones with triangular and square waves as compared to sinusoidal waves, there is a large difference experienced between experimental and numerical data. The reasons for this variation is not extremely clear. Furthermore, it is evident that there is a good visual agreement of both experimental and numerical deformed shape for the cones as exemplified in Figure 10 for the case of perfect cone and imperfect cones with $\mathrm{N}=12$. All cones fail through bulging in the region where the compressed load was applied. This failure mode is typical for axially compressed shells within the elastic plastic domain as reported in (Błachut and Ifayefunmi 2010b; Khakimova et al. 2016a; 2016b; Wagner, Hühne, and Khakimova 2019) for cones and (Evkin et al. 2019; Fan 2019) for cylinders.

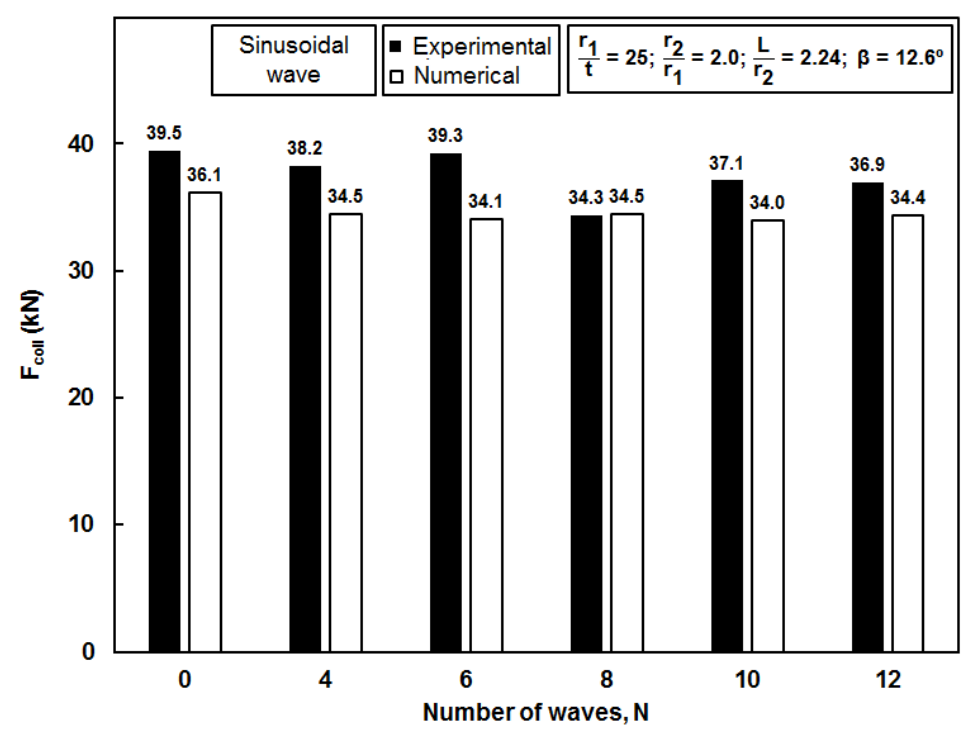

Figure 7. Comparison of average numerical and experimental plot of axial force versus number of waves for perfect and imperfect cones having sinusoidal wave. 


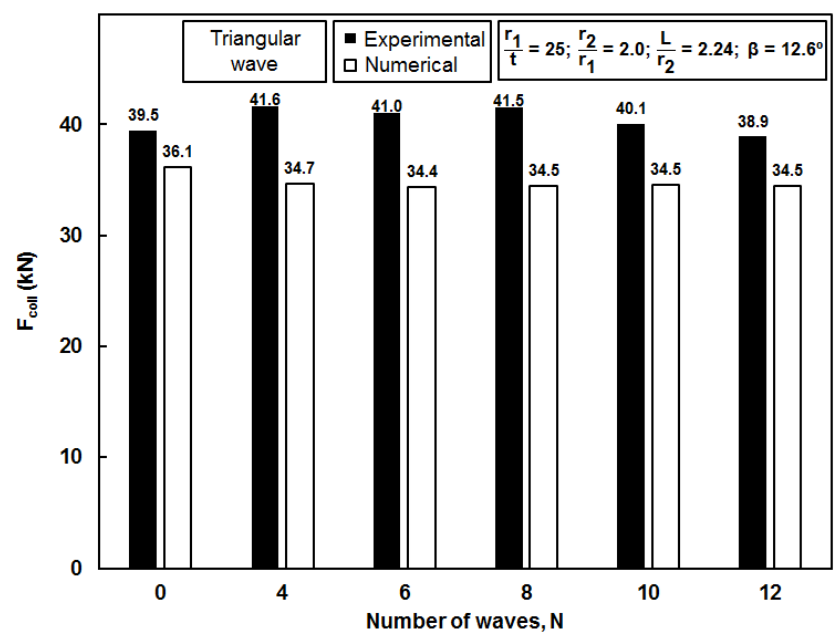

Figure 8. Comparison of average numerical and experimental plot of axial force number of waves for perfect and imperfect cones having triangular wave.

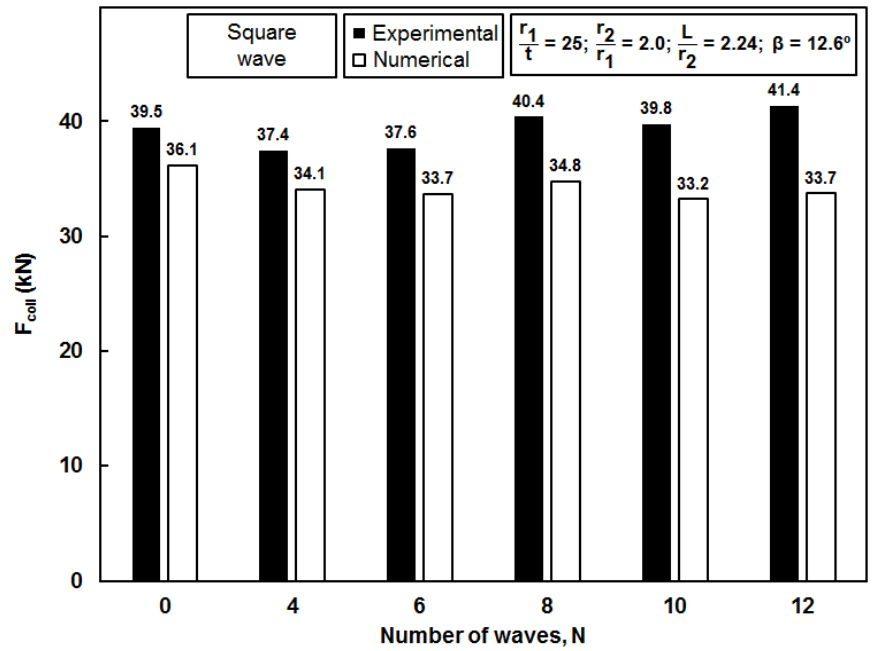

Figure 9. Comparison of average numerical and experimental plot of axial force versus number of waves for perfect and imperfect cones having square wave.

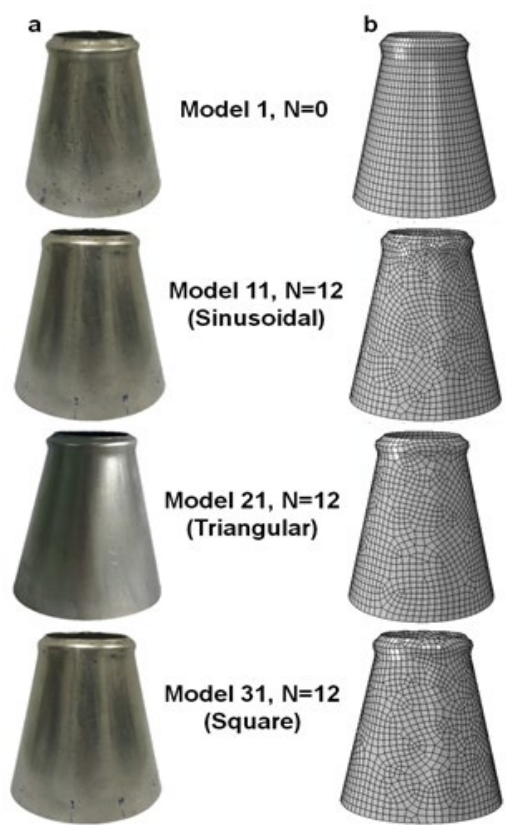

Figure 10. Typical view of deformed perfect and imperfect cones with $A=0.28$ (a) experimental and (b) numerical. 


\subsection{Further numerical calculation}

Further numerical computations were conducted to understand the (i) effect of imperfection amplitude, (ii) effect of wave number, (iii) effect of wave shape, (iv) effect of contact problems, and (v) effect of different type of imperfection on the buckling behaviour of imperfect truncated mild steel cone having uneven length subjected to axial compression. Conical models geometries, loading and boundary conditions are the same as discussed in section 3 - numerical modelling. For the numerical calculation in this section, nominal thickness of $1 \mathrm{~mm}$, axial imperfection amplitude, A, varied from $0-2.8$ and six different wave numbers, $N=2,4,6,8,10$ and 12 were considered. It is worth mentioning that for experimental programme in this paper, cones with wave number, $N=2$, was not tested. Three types of wave profiles were investigated, they are: (i) sinusoidal waves, (ii) triangular waves and (iii) square waves.

Firstly, computation of buckling load was carried out for perfect and imperfect conical model having sinusoidal wave number $(\mathrm{N}=0,2,4,6,8,10,12)$ with small radius-to-thickness ratio, $r_{1} / t=25$. The imperfection amplitude, $A$, was varied from $0-2.8$ and a constant nominal thickness of $1 \mathrm{~mm}$ was used. Figure 11 depicts the plot of collapse force against the number of sinusoidal waves. The collapse force is normalized by the collapse force for the perfect cone. The corresponding magnitude of the collapse force is given in Table 4. From Figure 11, it can be observed that the buckling loads of conical shell with non-uniform axial length is strongly dependent on the imperfection amplitude of the sinusoidal wave. It is apparent that as the imperfection amplitude increases, the buckling load of the conical shells decreases. As an example, imperfect conical shell with sinusoidal waves, $\mathrm{N}=2$, having imperfection amplitude, $A$, of 1.12 can only withstand $77 \%$ of the buckling load of the perfect cone as compare to cone with imperfection amplitude, A, of 0.28 , where the imperfect cone can sustain $92 \%$ of the buckling load of the perfect cone. However, increasing the number of waves seems to have minimal influence on the buckling load of the imperfect cone. For instance, at small imperfection amplitude, $\mathrm{A} \leq 0.28$, the lowest buckling load was observed at wave number, $\mathrm{N}=10$. And as the imperfection amplitudes increases, i.e., $0.56 \leq A<2.8$, the lowest buckling load was noticed at $N=4$, whilst for $A \geq 2.8$, the lowest buckling load was seen to occur at $\mathrm{N}=2$. This trend appears to be consistent with that of experiment presented in this paper. It is worth mentioning that the drop in numerical buckling load at $\mathrm{N}=8$ and $\mathrm{N}=10$ are very close. Hence, confirming the experimental prediction of $\mathrm{N}=8$.

Figure 12 presents the plot of collapse load against axial shortening for perfect and imperfect cone having four sinusoidal waves with imperfection amplitude, $A$ of 1.12 (point ' $P$ ' on Figure 11). In addition, finite element analysis was carried out to investigate the buckling resistance of imperfect cone with $N=4$ and $A=1.12$ having radius-to-thickness ratios of $r_{1} / t=100$. Figure 13 depicts the plot of collapse load versus axial shortening for imperfect cone with $N=4$ and $A=1.12$ with radius-to-thickness ratios of $r_{1} / t=100$. The corresponding magnitude of collapse load can be found in Table 5 . It is obvious that increasing the radius-to-thickness ratio of the cone results in increase in the cone sensitivity to imperfect length. For instance, cone with $A=1.12$, increasing the radius-to-thickness ratio from 25 to 100, the ratio of the load carrying capacity of the imperfect cone to their corresponding perfect cone is reduced from $75 \%$ to $34 \%$. Similarly, for cone with $A=2.24$, increasing the radius-to-thickness ratio from 25 to 100, the ratio of the load carrying capacity of the imperfect cone to their corresponding perfect cone is reduced from $61 \%$ to $31 \%$. From Figure 12, it can be seen that the load deflection curve for the perfect cone is linear up to the collapse load and afterward, a gentle fall in the post-collapse region. However, for the imperfect cone, the plot shows a wavy behaviour in the load deflection curve. This behaviour can be said to be as a result of the wavy nature of the compressed top edge of the cone. Although, for the perfect cone with $r_{1} / t=100$, there is a sharp drop in the curve within the post-collapse region. In addition, for the imperfect cone with $r_{1} / t=100$, the waviness of the load deflection curve is more pronounced as compared to imperfect cone with $r_{1} / t=25$.

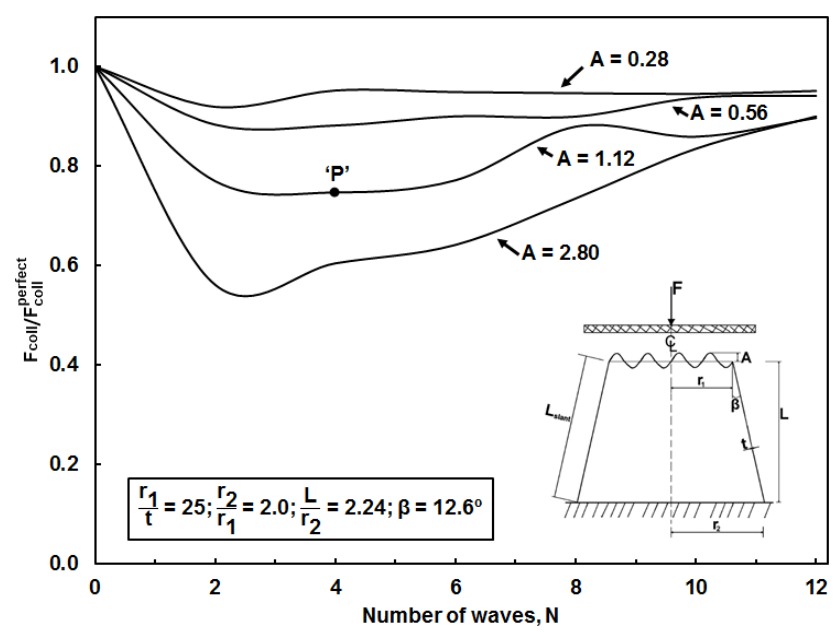

Figure 11. Effect of cone axial imperfection amplitude, A, on the buckling load of axially compressed imperfect cone having sinusoidal waves with small radius-to-thickness ratio, $r_{1} / t$ of 25 . 
Table 4. The buckling load for imperfect cone with different number of sinusoidal waves and axial imperfection amplitude-tothickness ratio ranging from $0-2.8$.

\begin{tabular}{|c|c|c|c|c|c|c|}
\hline \multirow{2}{*}{$\mathbf{N}$} & \multicolumn{6}{|c|}{ Collapse load $(k N)$ for different $A(t=1 \mathrm{~mm})$} \\
\hline & 0.28 & 0.56 & 1.12 & 1.68 & 2.24 & 2.8 \\
\hline 0 & 37.85 & 37.85 & 37.85 & 37.85 & 37.85 & 37.85 \\
\hline 2 & 34.8 & 33.44 & 29.14 & 25.22 & 23.29 & 21.25 \\
\hline 4 & 36.05 & 33.38 & 28.28 & 25.20 & 23.05 & 22.89 \\
\hline 6 & 35.92 & 34.07 & 29.21 & 27.07 & 25.52 & 24.29 \\
\hline 8 & 35.84 & 34.05 & 33.3 & 28.39 & 27.63 & 27.83 \\
\hline 10 & 35.78 & 35.49 & 32.52 & 30.59 & 31.01 & 31.61 \\
\hline 12 & 36.01 & 35.63 & 33.95 & 33.84 & 33.83 & 34.07 \\
\hline
\end{tabular}

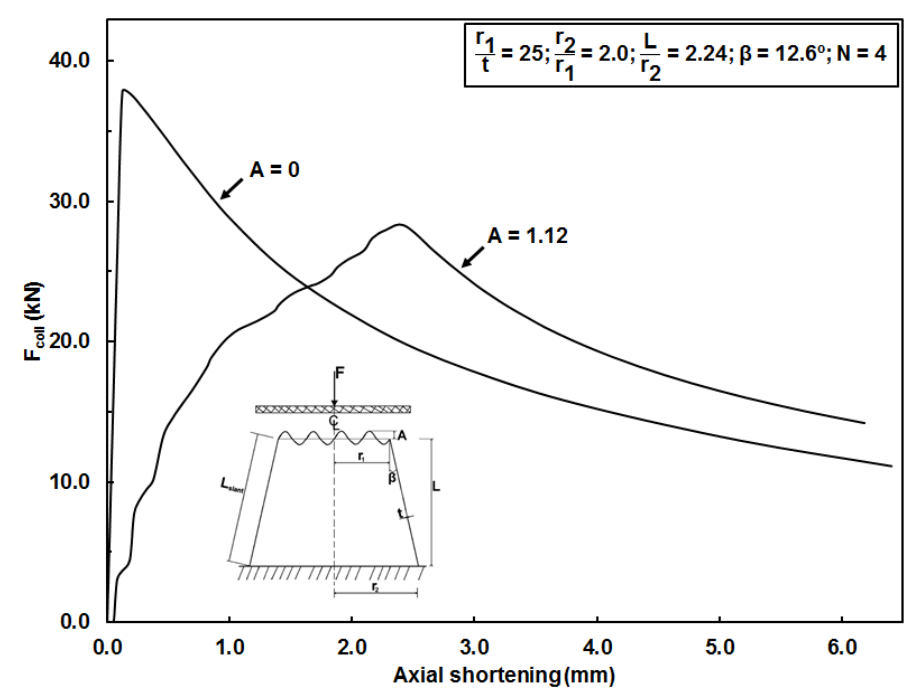

Figure 12. Plot of load versus compression extension for perfect and imperfect cone having radius-to-thickness ratio, $r_{1} / t=25$, with imperfection amplitude, $A=1.12$, and fixed number of wave at the top edge, $N=4$.

Table 5. Effect of imperfection amplitude and radius-to-thickness ratio on the buckling load of axial compressed uneven cone. The number in bracket is the ratio of buckling load of imperfect cone to perfect cone.

\begin{tabular}{ccccc}
\hline \multirow{2}{*}{$\mathbf{r} 1 / \mathbf{t}$} & $\mathbf{N}$ & $\mathbf{0}$ & \multicolumn{3}{c}{ Collapse load $(\mathbf{k N})$ for different A $(\mathbf{t}=\mathbf{1} \mathbf{~ m m})$} \\
\cline { 3 - 5 } & & 4 & $\mathbf{1 . 1 2}$ & $\mathbf{2 . 2 4}$ \\
\hline 25 & 4 & $9.182(1.0)$ & $28.282(0.75)$ & $23.052(0.61)$ \\
100 & & & $3.130(0.34)$ & $2.849(0.31)$ \\
\hline
\end{tabular}

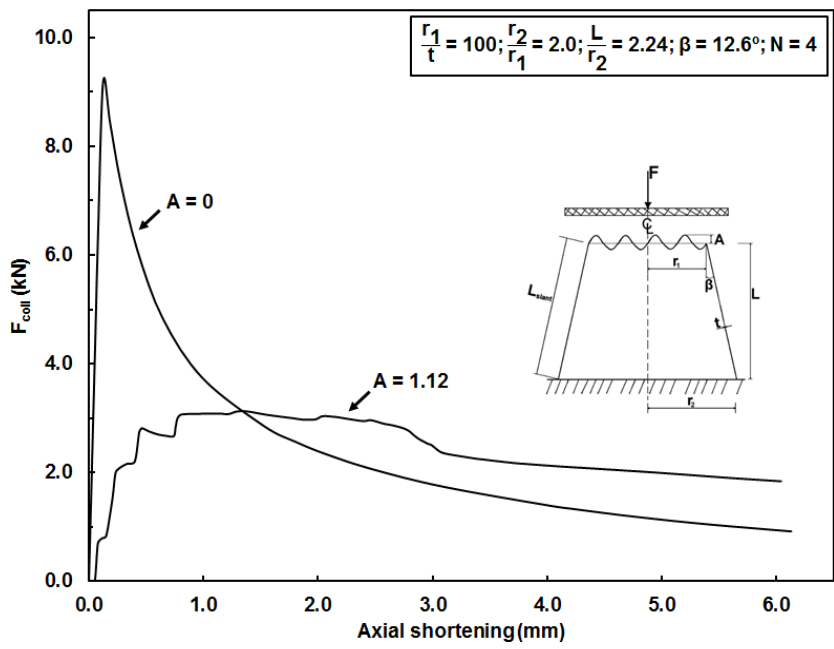

Figure 13. Plot of load versus compression extension for perfect and imperfect cone having radius-to-thickness ratio, $r_{1} / t=100$, with imperfection amplitude, $A=1.12$, and fixed number of wave at the top edge, $N=4$. 
Figures 14 and 15 depict the plot of collapse force against the number of waves for cones with triangular and square waves, respectively. The corresponding magnitude of collapse load are given in Table 6. Again from 14, it is obvious that increasing the axial imperfection amplitude will result in a decrease in the load carrying capacity of the conical structures. However, this is only true for square waves at small number of waves, as the wave number increases, the lines are seen to be crossing each other. Again, this behavior was similar to that obtained from experiment as can be seen in Figure 6 .

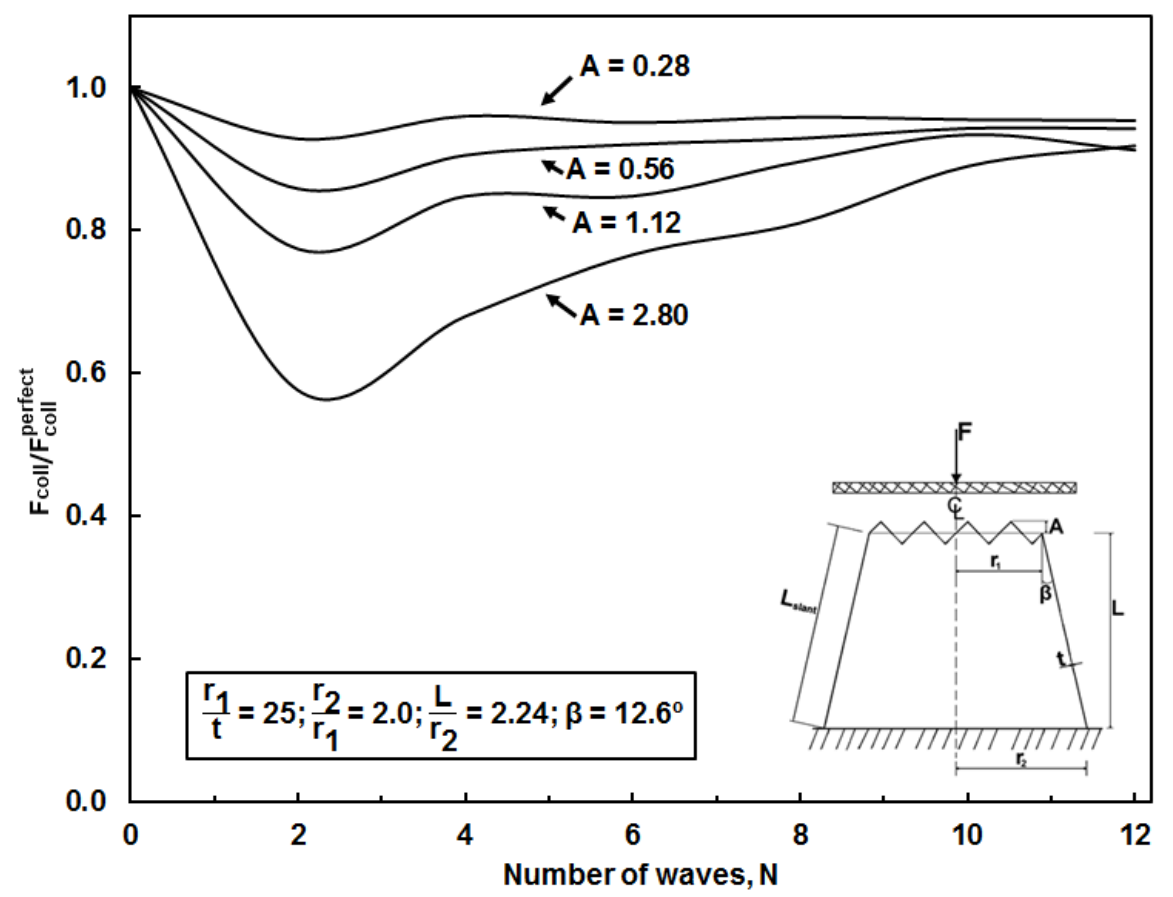

Figure 14. Effect of cone axial imperfection amplitude, A, on the buckling load of axially compressed imperfect cone having triangular waves with small radius-to-thickness ratio, $r_{1} / t$ of 25 .

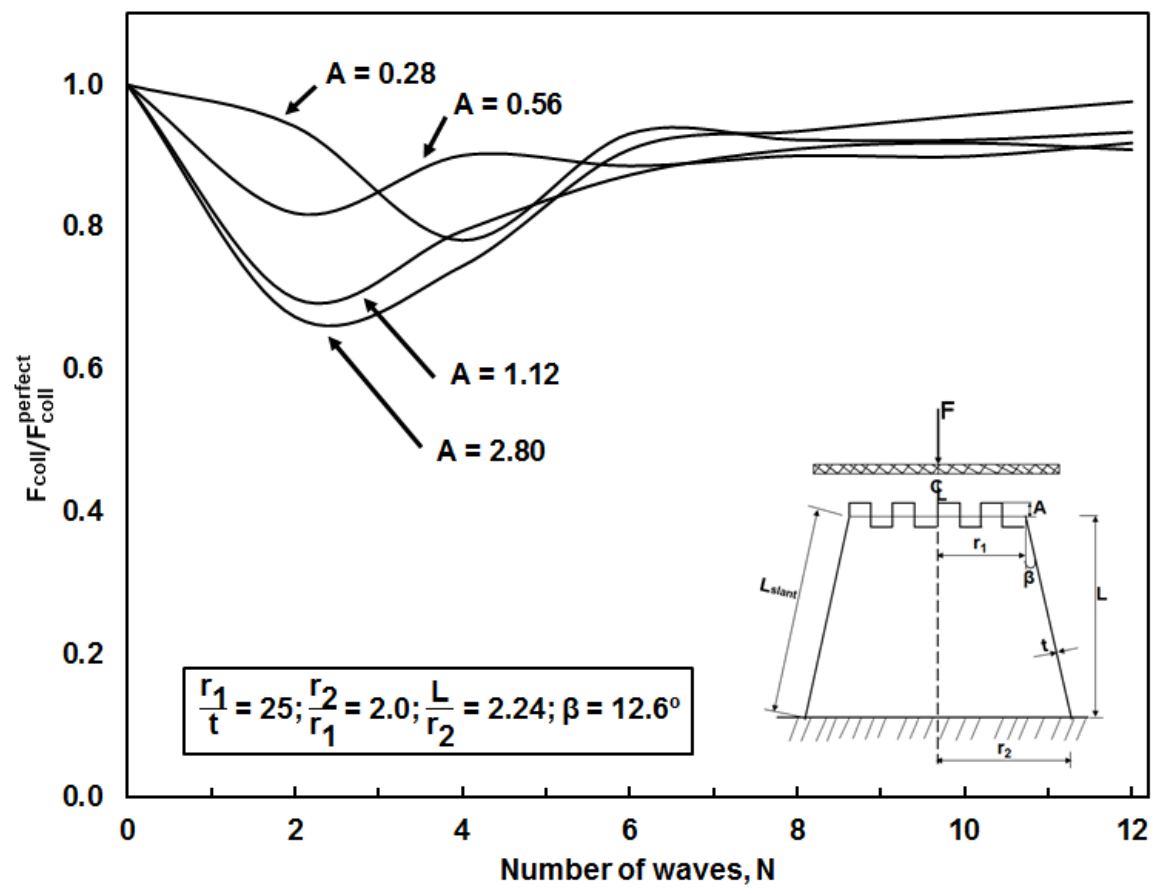

Figure 15. Effect of cone axial imperfection amplitude, A, on the buckling load of axially compressed imperfect cone having square waves with small radius-to-thickness ratio, $r_{1} / t$ of 25 . 
Table 6. The influence of wave amplitude, wave number and wave shape on the buckling load for imperfect cone with uneven length. (I) $\equiv$ sinusoidal waves, (II) $\equiv$ triangular waves, and (III) $\equiv$ square waves.

\begin{tabular}{|c|c|c|c|c|c|c|c|}
\hline \multirow{2}{*}{ Wave amplitude, A } & \multirow{2}{*}{ Wave Shape } & \multicolumn{6}{|c|}{ Collapse load (kN) for different wave number, $N, t=1$} \\
\hline & & 2 & 4 & 6 & 8 & 10 & 12 \\
\hline \multirow[t]{3}{*}{0.28} & (I) & 34.80 & 36.05 & 35.92 & 35.84 & 35.78 & 36.01 \\
\hline & (II) & 35.14 & 36.34 & 36.00 & 36.29 & 36.16 & 36.12 \\
\hline & (III) & 35.64 & 29.58 & 35.25 & 34.92 & 34.91 & 35.33 \\
\hline \multirow[t]{3}{*}{0.56} & ( & 33.44 & 33.38 & 34.07 & 34.05 & 35.49 & 35.63 \\
\hline & (II) & 32.50 & 34.27 & 34.84 & 35.16 & 35.70 & 35.68 \\
\hline & (III) & 31.02 & 34.09 & 33.54 & 34.08 & 34.04 & 34.76 \\
\hline \multirow[t]{3}{*}{1.12} & (I) & 29.14 & 28.28 & 29.21 & 33.30 & 32.52 & 33.95 \\
\hline & (II) & 29.31 & 32.10 & 32.10 & 33.94 & 35.35 & 34.55 \\
\hline & (III) & 26.49 & 30.08 & 33.06 & 34.44 & 34.76 & 34.40 \\
\hline \multirow[t]{3}{*}{1.68} & (I) & 25.22 & 25.20 & 27.07 & 28.39 & 30.59 & 33.84 \\
\hline & (II) & 26.72 & 30.29 & 31.18 & 32.85 & 33.76 & 34.70 \\
\hline & (III) & 26.02 & 27.50 & 33.88 & 34.74 & 35.42 & 33.69 \\
\hline \multirow[t]{3}{*}{2.24} & (I) & 23.29 & 23.05 & 25.52 & 27.63 & 31.01 & 33.83 \\
\hline & (II) & 24.39 & 27.93 & 29.96 & 31.78 & 34.19 & 35.48 \\
\hline & (III) & 25.81 & 27.56 & 33.52 & 33.76 & 36.06 & 36.25 \\
\hline \multirow[t]{3}{*}{2.8} & (I) & 21.25 & 22.89 & 24.29 & 27.83 & 31.61 & 34.07 \\
\hline & (II) & 21.81 & 25.73 & 29.00 & 30.69 & 33.69 & 34.78 \\
\hline & (III) & 25.51 & 28.21 & 34.42 & 35.37 & 36.22 & 36.96 \\
\hline
\end{tabular}

Secondly, the paper examines the influence of wave number, $\mathrm{N}$, on the load carrying capacity of the conical models. Figures 16-18 present the plot of axial collapse force against axial imperfection amplitude for cones with sinusoidal, triangular and square waves, respectively. Again, the corresponding magnitude of axial collapse load can be found in Table 6. It can be seen that small number of waves is sufficient to produce the largest reduction in the load carrying capacity of axially compressed conical shells especially at large imperfection amplitude. For conical model having sinusoidal wave and imperfection amplitude of 0.28 , the largest reduction in collapse load was observed at $\mathrm{N}=2$, followed by $\mathrm{N}=10$ (see Figure 16), whereas, for conical models with triangular waves having the same imperfection amplitude, the largest drop in collapse load was seen to be found at $N=2$, followed by $N=12$ (see Figure 17), and for conical model with square waves with the same imperfection amplitude, the lowest buckling load was noticed at $\mathrm{N}=4$ (see Figure 18). Again, this result is the same with that of experiment as presented in Figure 6. In general, it can be said that increasing the waves number on the cones has minimal effect on the buckling load as reported in (Błachut 2015) for cylinder with sinusoidal waves.

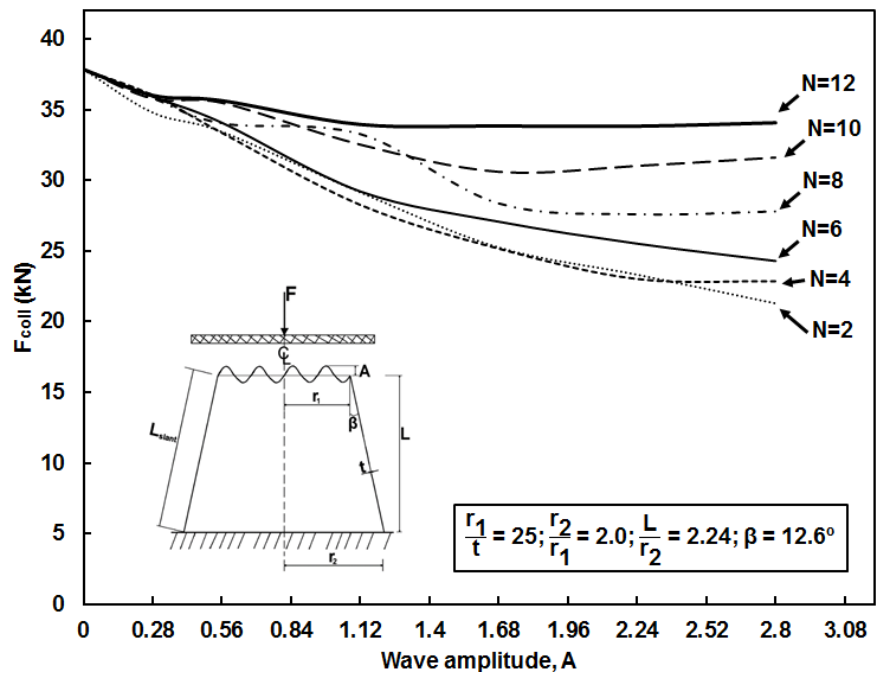

Figure 16. Effect of wave number, $\mathrm{N}$, on the buckling load of axially compressed imperfect cone having sinusoidal waves with small radius-to-thickness ratio, $\mathrm{r}_{1} / \mathrm{t}$ of 25 . 


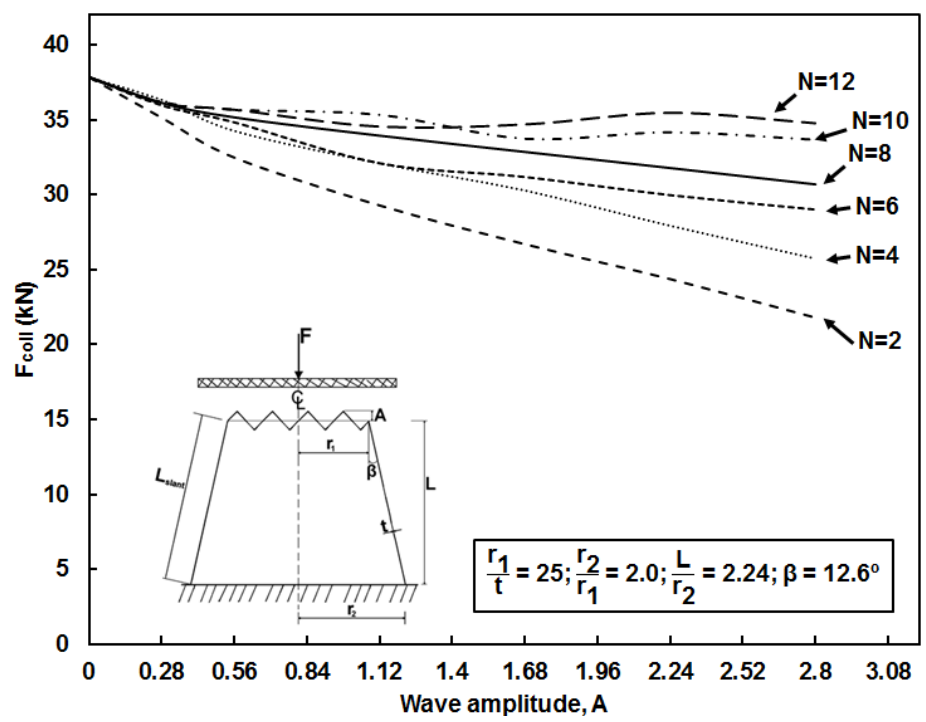

Figure 17. Effect of wave number, $\mathrm{N}$, on the buckling load of axially compressed imperfect cone having triangular waves with small radius-to-thickness ratio, $\mathrm{r}_{1} / \mathrm{t}$ of 25 .

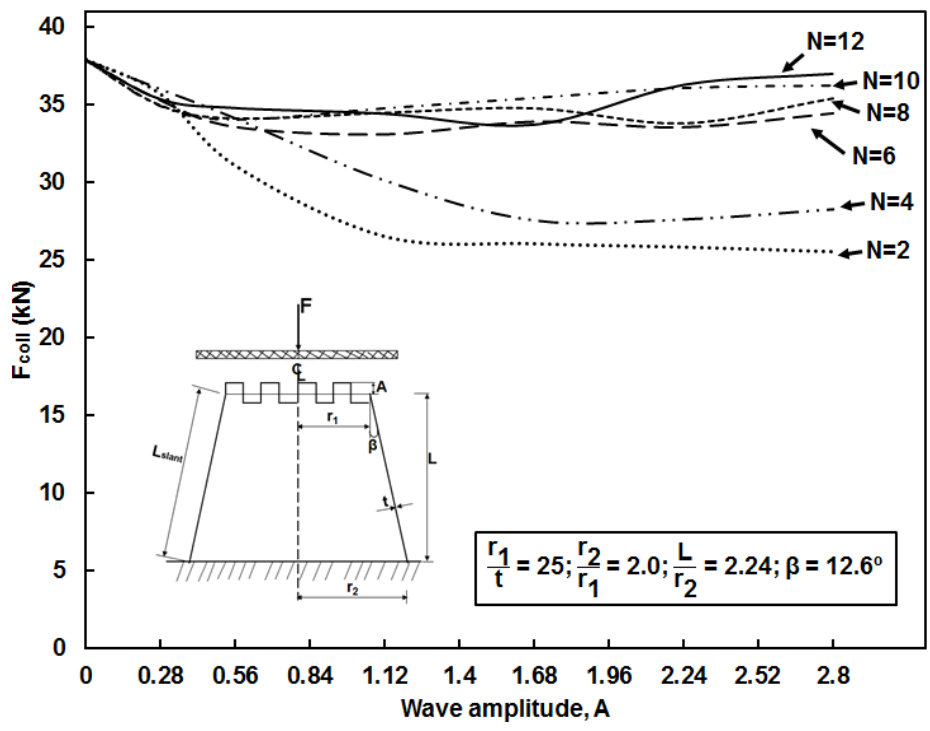

Figure 18. Effect of wave number, $\mathrm{N}$, on the buckling load of axially compressed imperfect cone having square waves with small radius-to-thickness ratio, $r_{1} / t$ of 25 .

Thirdly, the paper examines the influence of imperfection shape on the load carrying capacity of the conical models. Three types of initial geometric imperfection shape were considered, they are: (i) sinusoidal waves, (ii) triangular waves, and (iii) square waves. Figures 19-21 present the plot of axial collapse force against axial imperfection amplitude for cones with different waves numbers, $\mathrm{N}=4,6$ and 8 , respectively, with the corresponding magnitude of collapse load given in Table 6. Observed here for all the different wave number considered, at small imperfection amplitude, $\mathrm{A} \leq 0.56$, cone with triangular waves have the least imperfection sensitivity. This may be said to be due to the fact that for the triangular waves, the shape helps to spread out the load, thereby being able to sustain more load at the compressed edge of cone as argued in (Ifayefunmi and Zulkefli 2019) for cylindrical shells. In general, the largest reduction in buckling load is observed for cone with square wave. This confirms the results from experimental tests discussed earlier in the paper (see Figure 6). However, from experiment, for wave number, $\mathrm{N}=8$, the largest drop in buckling load was found to be cone with sinusoidal waves. This can be attributed to the use of nominal thickness value in the numerical analysis. It is hoped that using measured thickness will produce the same result as the experiments as exemplified in column 3 of Table 2. Furthermore, at relatively large imperfection amplitude, $1.12 \leq \mathrm{A} \leq 2.8$, except for $N=4$, where cone with triangular waves produce the least imperfection up until $(A=2.24)$, the square waves was the least sensitive for all the number of waves considered. Here, the largest reduction in the load carrying capacity of the structures was observed for conical shells with sinusoidal waves. 


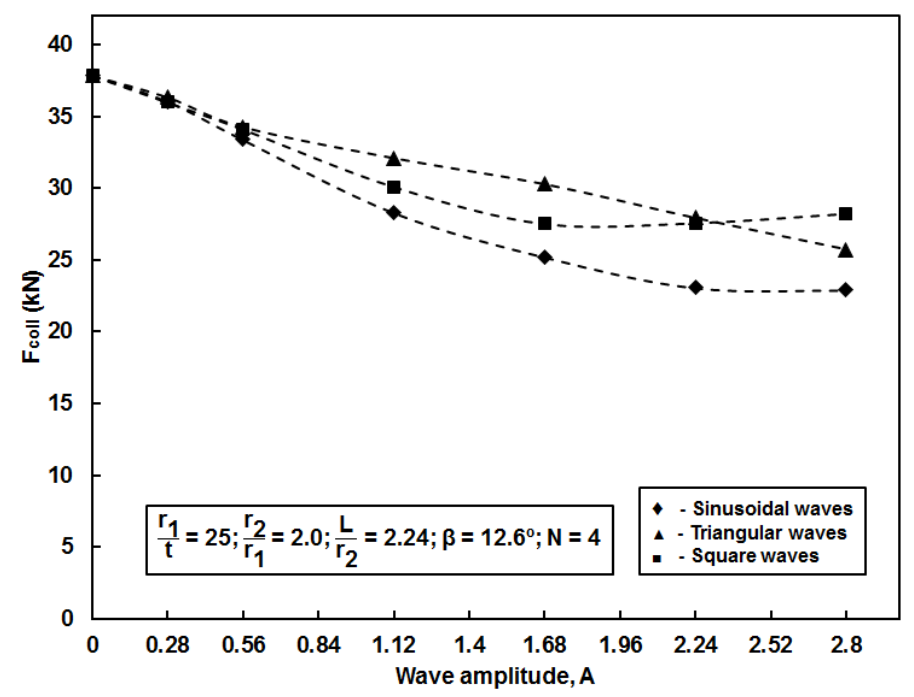

Figure 19. Effect of wave shape on the buckling load of axially compressed imperfect cone having waves number, $N=4$, with small radius-to-thickness ratio, $r_{1} / t$ of 25 .

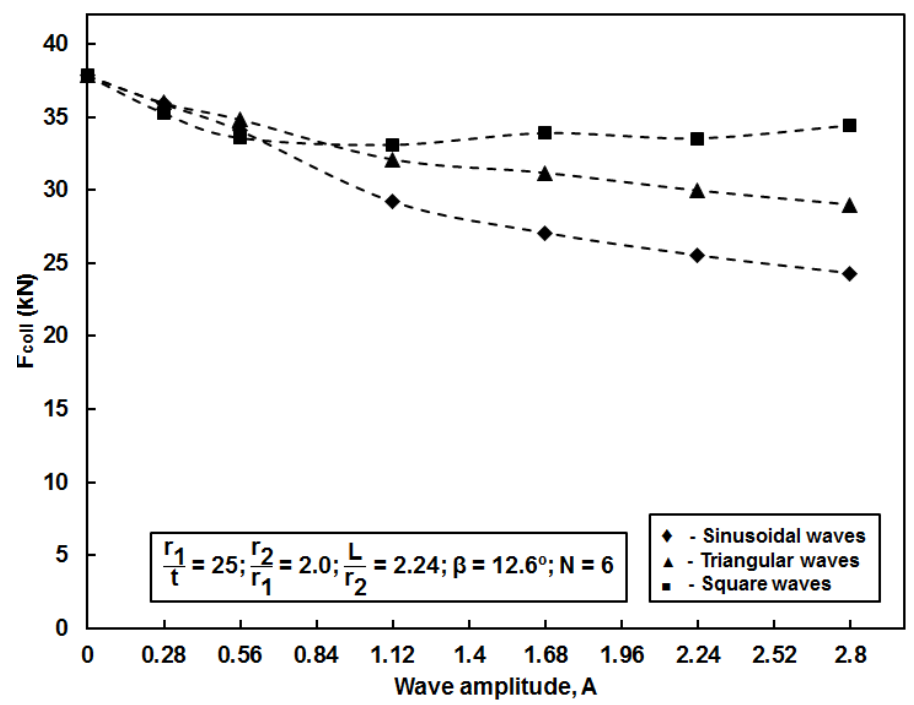

Figure 20. Effect of wave shape on the buckling load of axially compressed imperfect cone having waves number, $N=6$, with small radius-to-thickness ratio, $r_{1} / t$ of 25 .

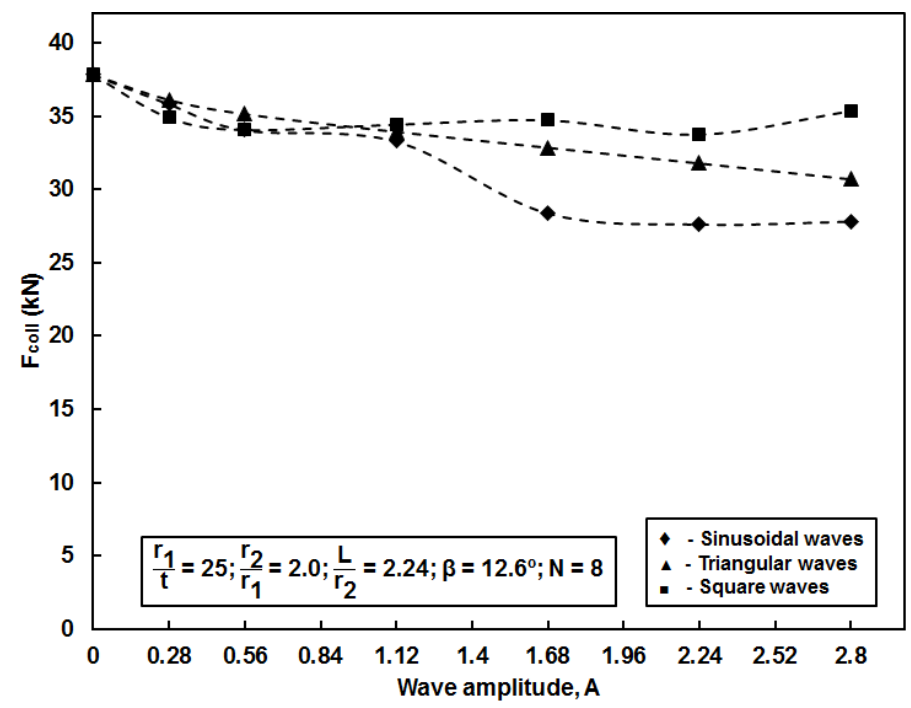

Figure 21. Effect of wave shape on the buckling load of axially compressed imperfect cone having waves number, $N=8$, with small radius-to-thickness ratio, $r_{1} / t$ of 25 . 
Fourthly, the contact interaction problem between the imperfect cone having sinusoidal waves with imperfection amplitude, A, ranging from 0.28 to 2.80 . Two different contact interaction between the rigid plate and the compressed edge of the cone were analyzed, and they are: (i) contact interaction with set of top edge nodes, and (ii) contact interaction with set of $\mathrm{N}$-point nodes. Table 7 gives the result obtained from the ensuing numerical computations. Surprisingly, the contact interaction between the rigid plate and the deformable cone has a significant influence on the collapse load of the imperfect cone. This implies that the choice of contact interaction employed during the analysis is very important. However, experiment reveals that this choice strongly depends on the imperfection amplitude as reported in (Ifayefunmi, Mahidan and Wang 2019), where for small amplitude, the buckling of the cone was experienced and at large amplitude, local buckling of the waves was observed. To further explore the contact interaction problem, growth of contact area during buckling process was investigated. Five points were chosen to examine the contact interaction between the rigid plate and the deformable cone at loading steps. The points are represented by ' $i$ ' to ' $v$ ', where points ' $\mathrm{i}$ ' to 'iii' are within the pre-collapse region, point 'iv' refers to the collapse point of the cone and point ' $\mathrm{V}$ ' is within the post-collapse region. Figure 22 presents growth of contact area on five chosen points during the buckling stages for a conical shell having sinusoidal waves, $\mathrm{N}=4$, and imperfection amplitude, $\mathrm{A}$, ranging from 0.28 to 2.8 . The horizontal lines indicate the size of the contact area at the chosen point.

From Figure 22a, it can be noticed that full contact was recorded for the cone at collapse $(F=36.0455 \mathrm{kN})$. The contact area recorded at all four points of the cone are the same, indicating the maximum length of conical shell. Full contact is also noticed in the post-collapse region represented by point ' $v$ '. Similar trend was observed for cones with imperfection amplitude, $A=0.56$ (see Figure $22 \mathrm{~b}$ ). This result is somewhat similar to what was reported for cylinder in (Błachut 2010). In the contrary, for cones with large amplitude i.e., $1.12 \leq A \leq 2.8$, it is seen that contact of the entire area was not achieved at collapse. Full contact was only achieved at the post collapse path (point ' $v$ '). Hence, it can be said that the contact interaction between the rigid plate and the deformable cone is largely influenced by imperfection amplitude. It was found that for cone with smaller imperfection amplitude, the buckling is happening at the top edge nodes which is characterized by the buckling of the cone - as seen for $(A=0.28)$ in Figure 5 of (Ifayefunmi, Mahidan and Wang 2019). Whilst, for cone having higher imperfection amplitude, the buckling will happen at N-point nodes which denotes localized buckling of the waves - as seen for $(A=2.8)$ in Figure 6 of (Ifayefunmi, Mahidan and Wang 2019). The corresponding load deflection curve of the cones indicating the location of the five point is presented in Figure 23 (for cone having imperfection amplitude, $A=0.28$ ) and Figure 24 (for cone having imperfection amplitude, $A=2.8$ ).

Table 7. The effect of contact interaction on the buckling load for imperfect cone with different number of sinusoidal waves and axial imperfection amplitude. Note: $(\mathrm{I}) \equiv$ contact interaction with set of top edge nodes, and (II) $\equiv$ contact interaction with set of $\mathrm{N}$ point nodes.

\begin{tabular}{|c|c|c|c|c|c|c|c|c|c|c|c|c|}
\hline \multirow{3}{*}{$\mathbf{N}$} & \multicolumn{12}{|c|}{ Collapse load $(k N)$ for different $A(t=1 \mathrm{~mm})$} \\
\hline & \multicolumn{2}{|c|}{0.28} & \multicolumn{2}{|c|}{0.56} & \multicolumn{2}{|c|}{1.12} & \multicolumn{2}{|c|}{1.68} & \multicolumn{2}{|c|}{2.24} & \multicolumn{2}{|c|}{2.8} \\
\hline & 1 & II & 1 & II & 1 & II & 1 & II & 1 & II & 1 & II \\
\hline 0 & 37.851 & 37.851 & 37.851 & 37.851 & 37.851 & 37.851 & 37.851 & 37.851 & 37.851 & 37.851 & 37.851 & 37.851 \\
\hline 2 & 34.795 & 6.085 & 33.438 & 6.269 & 29.137 & 5.676 & 25.222 & 5.293 & 23.293 & 5.78 & 21.252 & 5.547 \\
\hline 4 & 36.046 & 11.901 & 33.376 & 11.421 & 28.282 & 9.014 & 25.197 & 9.147 & 23.052 & 9.573 & 22.889 & 8.675 \\
\hline 6 & 35.917 & 16.434 & 34.072 & 16.114 & 29.209 & 14.817 & 27.07 & 13.816 & 25.518 & 14.252 & 24.292 & 13.508 \\
\hline 8 & 35.837 & 19.829 & 34.053 & 19.543 & 33.295 & 20.725 & 28.388 & 15.321 & 27.627 & 14.3 & 27.83 & 12.266 \\
\hline 10 & 35.783 & 24.667 & 35.494 & 24.237 & 32.522 & 18.53 & 30.591 & 16.193 & 31.01 & 13.857 & 31.607 & 13.442 \\
\hline 12 & 36.007 & 26.991 & 35.634 & 26.026 & 33.949 & 20.305 & 33.844 & 19.241 & 33.829 & 14.564 & 34.069 & 14.031 \\
\hline
\end{tabular}

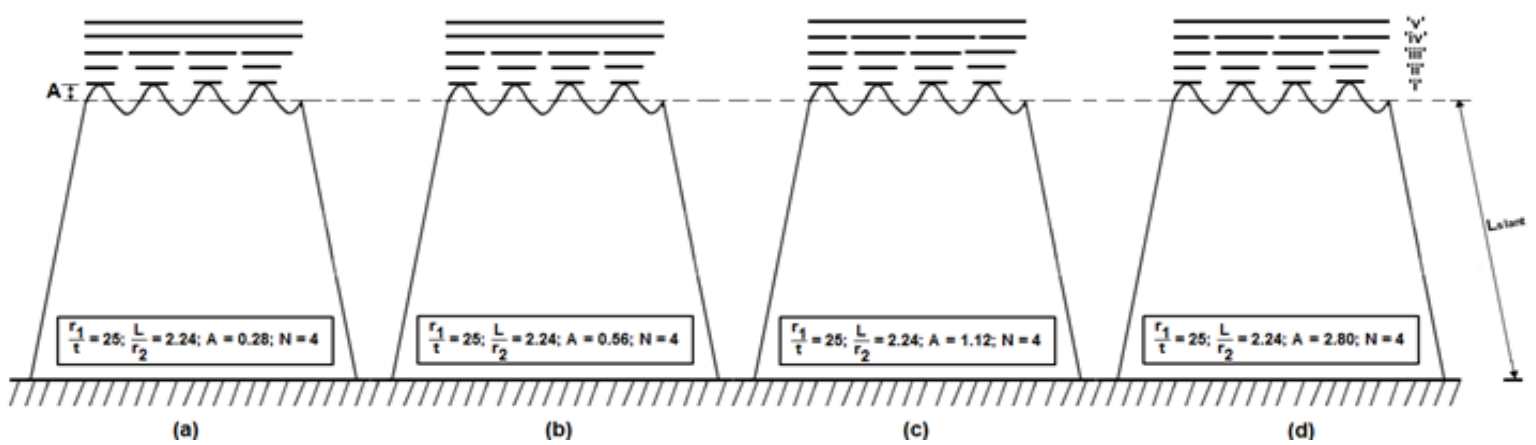

Figure 22. Growth of contact area on five chosen points on the loading steps for a conical shell having sinusoidal waves $(\mathrm{N}=4)$. 


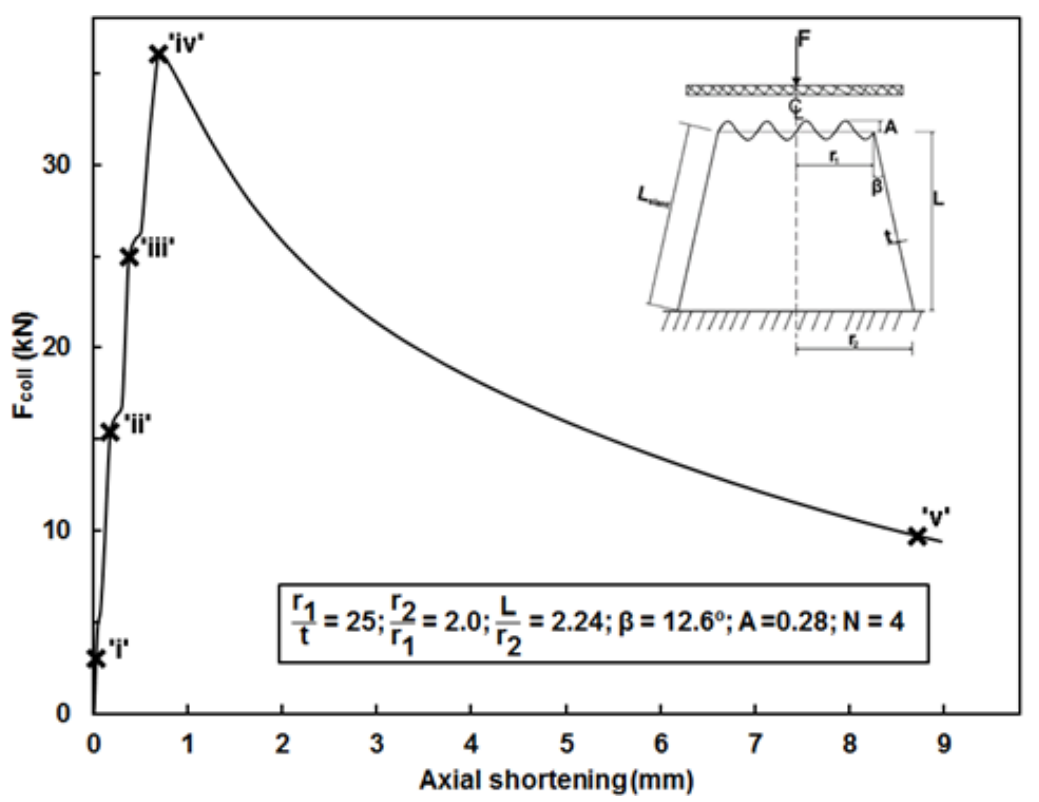

Figure 23. Plot of collapse load versus axial shortening for cone having sinusoidal waves with $\mathrm{N}=4$ and $\mathrm{A}=0.28$, indicating the location of the contact area.

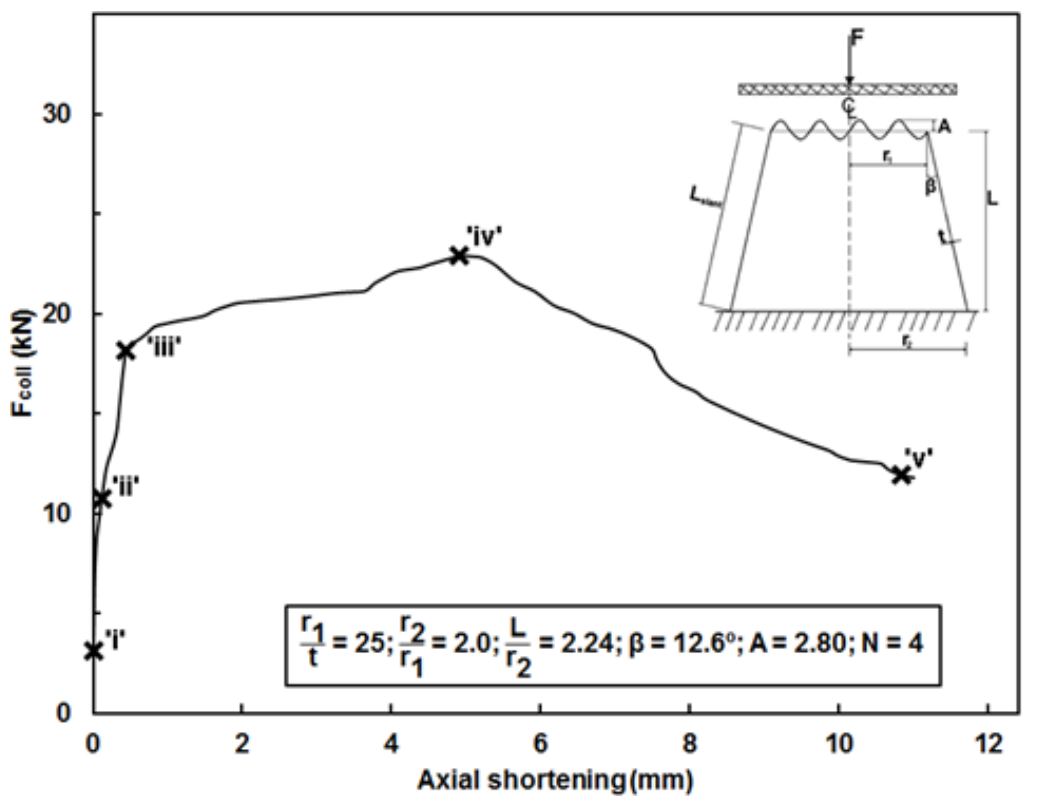

Figure 24. Plot of collapse load versus axial shortening for cone having sinusoidal waves with $N=4$ and $A=2.80$, indicating the location of the contact area.

Lastly, comparison of imperfection sensitivity for axially compressed cones with uneven length imperfection and eigenmode imperfection was investigated. It is a general believe that different types of imperfections will affect the magnitude of load carrying capacity of thin-walled structures such as conical shells. The eigenmode imperfection is one of the most commonly used imperfection type in thin-walled structures. In this analysis, the eigenmodes were obtained by running eigenvalue buckling using subspace solver. A total of ten eigenmodes were considered in the finite element analysis. The modes were imposed on the perfect conical shell geometry to study its effect towards the buckling of cones. Several eigenmodes shape is a necessity when determining the shell's worst sensitivity towards eigenmode imperfection. The imperfection amplitude is kept the same for comparison purposes to be: $0 \leq A \leq 2.80$. Figure 25 depicts the plot of comparison of sensitivity of conical shells to four different imperfection type, i.e., uneven length with sinusoidal waves, uneven length with triangular waves, uneven length with square waves and eigenmode imperfections. Surprisingly, it is obvious that axially compressed cones with uneven length produce the worst imperfection at small imperfection amplitude, $0 \leq A \leq 1.12$. However, as the imperfection amplitude gradually increases, eigenmode imperfection takes the worst case. This result is supported by the findings from (Błachut 2015) for axially compressed cylinders with sinusoidal 
uneven length. It must be stressed here that this finding is very significant since the imperfection amplitude encountered in real life application are within the range $0 \leq A \leq 1.12$. This results highlight the severity of uneven length imperfection on the buckling behavior of axially compressed cones which will be of high importance for design purpose in order to prevent potential failure of the structure.

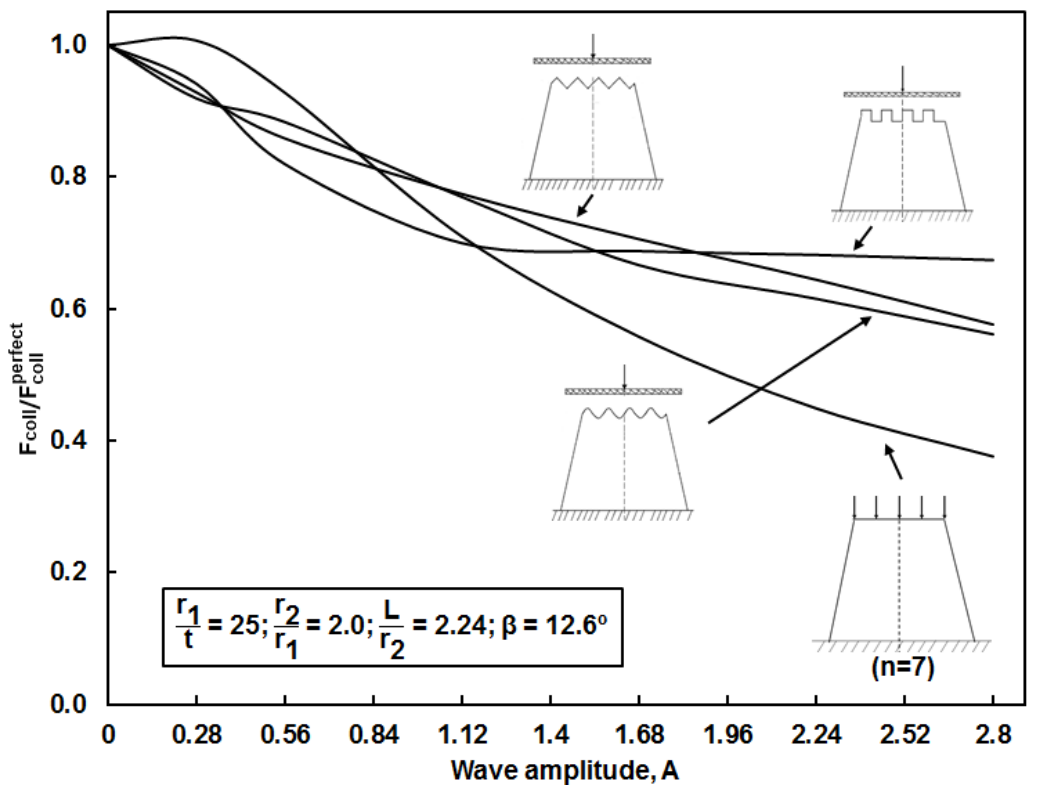

Figure 25. Plot of comparison of sensitivity of conical shells to four different imperfection type, i.e., uneven length with sinusoidal waves $(N=2)$, uneven length with triangular waves $(N=2)$, uneven length with square waves $(N=2)$ and eigenmode imperfection.

\section{CONCLUSION}

Axial compressive test results on thirty-two conical models with imperfect axial length manufactured in pairs having three different wave profiles and its accompanying numerical predictions were presented in this paper. Repeatability of experimental data was good. Also, experimental results are in good agreement with numerical predictions especially for conical shells having sinusoidal waves. From the results, it can be confirmed that imperfect axial length has a strong influence on the load carrying capacity of the conical geometry considered.

From the foregoing results, the following conclusions can be drawn: (i) it is apparent that imperfection amplitude plays a great role towards the buckling load of conical shells; as the imperfection amplitude increases, the buckling load of the conical shells decreases, (ii) increasing the waves number on the compressed edge of the cone has marginal effect on the load carrying capacity of conical shells, (iii) at small imperfection amplitude $(0<A \leq 0.56)$, cones with square waves produced the largest reduction in the load carrying capacity of the structures, while at larger imperfection amplitude $(1.12 \leq \mathrm{A} \leq 2.8)$, conical shells having sinusoidal waves result in the largest drop in the buckling load of the structures, (iv) the contact interaction between the rigid plate and the deformable cone has significant influence on the buckling strength of axially compressed cone having imperfect axial length and strongly depends on imperfection amplitude, A. The magnitude of imperfection amplitude determines where the buckling of the shell will occur either (a) the buckling on cones for small imperfection amplitude $(A<1.12)$ or $(b)$ localized buckling on waves for larger amplitude $(A \geq 1.12)$, and $(v)$ buckling load of conical shells with imperfect axial length are significantly lower in comparison the same conical shells having eigenmode imperfection. It is recommended that this comparison is extended to other types of geometrical imperfection to provide a comprehensive understanding on the imperfection sensitivity of conical shell structures.

\section{Acknowledgment}

The authors would like to acknowledge the financial support received from Universiti Teknikal Malaysia Melaka (UTeM) and the Ministry of Education Malaysia under Fundamental Research Grant Scheme FRGS/2018/FTKMPCARE/F00386. 
Author's Contribuitions: Conceptualization, O Ifayefunmi; Investigation, FM Mahidan; Formal analysis, FM Mahidan, O Ifayefunmi; Writing - original draft, FM Mahidan, O Ifayefunmi; Visualization, FM Mahidan, O Ifayefunmi; Writing - review \& editing, O Ifayefunmi, FM Mahidan; Funding acquisition, O Ifayefunmi; Supervision, $O$ Ifayefunmi.

Editor: Marcílio Alves.

\section{References}

Ali, D. (2013). Buckling of cracked conical frusta under axial compression. Research Journal of Recent Sciences 2(2):33-39.

Błachut, J. (2010). Buckling of axially compressed cylinders with imperfect length. Computers and Structures 88(5-6):365-74. https://doi.org/10.1016/j.compstruc.2009.11.010.

Błachut, J. (2012). Buckling of truncated cones with localized imperfections. American Society of Mechanical Engineers, Pressure Vessels and Piping Division (Publication) PVP 3:3-11. https://doi.org/10.1115/PVP2012-78374.

Błachut, J. (2013). Combined stability of geometrically imperfect conical shells. Thin-Walled Structures 67:121-28. https://doi.org/10.1016/j.tws.2013.02.007.

Błachut, J. (2015). Buckling of cylinders with imperfect length. Journal of Pressure Vessel Technology, Transactions of the ASME 137 (1):1-7. https://doi.org/10.1115/1.4027246.

Błachut, J., and Ifayefunmi, O. (2010a). Buckling of unstiffened steel cones subjected to axial compression and external pressure. Proceedings of the International Conference on Ocean, Offshore and Arctic Engineering, (June 6-11):385-398. New York, USA. (ISBN: 978-0-7918-4910-1).

Błachut, J., and Ifayefunmi, O. (2010b). Plastic buckling of conical shells. Journal of Offshore Mechanics and Arctic Engineering, 132(4): 041401-1-041401-11. https://doi.org/10.1115/1.4001437

Błachut, J., Ifayefunmi, O., and Corfa, M. (2011). Collapse and buckling of conical shells. Proceedings of the International Offshore and Polar Engineering Conference, (June 19-24):887-893. Maui, Hawaii, USA. (ISBN 978-1-880653-96-8).

Castro, S. G. P., Mittelstedt, C., Monteiro, F. A. C., Arbelo, M. A., Ziegmann, G., and Degenhardt, R. (2014). Linear buckling predictions of unstiffened laminated composite cylinders and cones under various loading and boundary conditions using semi-analytical models. Composite Structures, 118(1):303-315. https://doi.org/10.1016/j.compstruct.2014.07.037

Castro, S. G. P., Mittelstedt, C., Monteiro, F. A. C., Degenhardt, R., and Ziegmann, G. (2015). Evaluation of non-linear buckling loads of geometrically imperfect composite cylinders and cones with the Ritz method. Composite Structures, 122:284-299. https://doi.org/10.1016/j.compstruct.2014.11.050

Chryssanthopoulos, M. K., and Spagnoli, A. (1997). The influence of radial edge constraint on the stability of stiffened conical shells in compression. Thin-Walled Structures, 27(2):147-163. https://doi.org/10.1016/S0263-8231(96)00035-3

Evkin, A., Krasovsky, V., Lykhachova, O., and Marchenko, V. (2019). Local buckling of axially compressed cylindrical shells with different boundary conditions. Thin-Walled Structures, 141(April):374-388. https://doi.org/10.1016/j.tws.2019.04.039

Fan, H. (2019). Critical buckling load prediction of axially compressed cylindrical shell based on non-destructive probing method. Thin-Walled Structures 139 (March):91-104. https://doi.org/10.1016/j.tws.2019.02.034.

Goldfeld, Y. (2007). Imperfection sensitivity of laminated conical shells. International Journal of Solids and Structures 44 (3-4): 1221-41. https://doi.org/10.1016/j.ijsolstr.2006.06.016.

Goldfeld, Y., Sheinman, I., and Baruch, M. (2003). Imperfection sensitivity of conical shells. AIAA Journal, 41(3):517-524. https://doi.org/10.2514/2.1976

Goldfeld, Y., and Arbocz, J. (2004). Buckling of Laminated Conical Shells Given the Variations of the Stiffness Coefficients. AIAA Journal, 42(3):642-649. https://doi.org/10.2514/1.2765

Hibbit, Karlsson, and Sorensen Inc., 2006, ABAQUS - Theory and Standard User's Manual Version 6.3, Pawtucket, RI, 028604847, USA

Ifayefunmi, O. (2014). A survey of buckling of conical shells subjected to axial compression and external pressure. Journal of Engineering Science and Technology Review 7(2):182-89. https://doi.org/10.25103/jestr.072.27.

Ifayefunmi, O. (2017). Plastic buckling of conical shell with non-continuous edge support. International Journal of Mechanical and Mechatronics Engineering 17(1):143-52. 
Ifayefunmi, O., and Błachut, J. (2011). The effect of shape, thickness and boundary imperfections on plastic buckling of cones. Proceedings of the International Conference on Offshore Mechanics and Arctic Engineering - OMAE, 2(January):23-33. https://doi.org/10.1115/OMAE2011-49055

Ifayefunmi, O., and Błachut, J. (2018). Imperfection Sensitivity: A Review of Buckling Behavior of Cones, Cylinders, and Domes. Journal of Pressure Vessel Technology, Transactions of the ASME, 140(5):050801-1-8. https://doi.org/10.1115/1.4039695

Ifayefunmi, O., and Ibrahim, D. D. (2018). The effect of material discontinuity on the flanges of axially compressed steel cones. International Journal of Mechanical Engineering and Technology, 9(6):32-41. Retrieved from http://www.iaeme.com/IJMET/issues.asp?JType=IJMETandVType=9\&IType=6

Ifayefunmi, O., Kadmin, A. F., Chang, K. L., and Aziz, A. (2018). Influence of boundary condition on the buckling of axially compressed cones and cylinders. International Journal of Mechanical Engineering and Technology, 9(4):1106-1116.

Ifayefunmi, O., and Błachut, J. (2013). Instabilities in imperfect thick cones subjected to axial compression and external pressure. Marine Structures, 33:297-307. https://doi.org/10.1016/j.marstruc.2013.06.004

Ifayefunmi, O., Mahidan, F. M., and Maslan, M. H. (2020). Instability of Conical Shells with Multiple Dimples under Axial Compression. International Journal of Recent Technology and Engineering, 8(5):1022-1027. https://doi.org/10.35940/ijrte.e6102.018520

Ifayefunmi, O., Mahidan, F. M., and Wang, S. H. (2019). Buckling of cones with imperfect length subjected to axial compression. International Journal of Mechanical and Production Engineering Research and Development, 9(4):219-228.

Ifayefunmi, O., and Zulkefli, N.A. (2019). Buckling of cylinders with uneven length subjected to axial compression. Proceedings of the International Offshore (Ocean) and Polar Engineering Conference, ISOPE-2019, 4106-4114, Honolulu, Hawaii, USA (ISBN 978-1-880653-85-2)

Jabareen, M., and Sheinman, I. (2006). Postbuckling analysis of geometrically imperfect conical shells. Journal of Engineering Mechanics, 132(12):1326-1334. https://doi.org/10.1061/(ASCE)0733-9399(2006)132:12(1326)

Khakimova, R., Warren, C. J., Zimmermann, R., Castro, S. G. P., Arbelo, M. A., and Degenhardt, R. (2014). The single perturbation load approach applied to imperfection sensitive conical composite structures. Thin-Walled Structures, 84:369377. https://doi.org/10.1016/j.tws.2014.07.005

Khakimova, R., Wilckens, D., Reichardt, J., Zimmermann, R., and Degenhardt, R. (2016b). Buckling of axially compressed CFRP truncated cones: Experimental and numerical investigation. Composite Structures, 146:232-247. https://doi.org/10.1016/j.compstruct.2016.02.023

Khakimova, R., Zimmermann, R., Wilckens, D., Rohwer, K., and Degenhardt, R. (2016a). Buckling of axially compressed CFRP truncated cones with additional lateral load: Experimental and numerical investigation. Composite Structures, 157:436-447. https://doi.org/10.1016/j.compstruct.2016.08.011

Maali, M., Showkati, H., and Mahdi Fatemi, S. (2012). Investigation of the buckling behavior of conical shells under weldinduced imperfections. Thin-Walled Structures, 57:13-24. https://doi.org/10.1016/j.tws.2012.04.003

Mahidan, F. M., and Ifayefunmi, O. (2020). Further experiments on buckling of cones with imperfect length subjected to axial compression. International Journal of Mechanical and Production Engineering Research and Development, 10(2):65-76. https://doi.org/10.24247/ijmperdapr20206

Ning, X., and S. Pellegrino. (2013). Imperfection-insensitive axially loaded thin cylindrical shells. 54th AIAA/ASME/ASCE/AHS/ASC Structures, Structural Dynamics and Materials Conference, Boston, MA, AIAA-2013-1768

Ning, X., and Pellegrino, S. (2015). Imperfection-insensitive axially loaded thin cylindrical shells. International Journal of Solids and Structures, 62(June 2016):39-51. https://doi.org/10.1016/j.ijsolstr.2014.12.030

Ning, X., and Pellegrino, S. (2017). Experiments on imperfection insensitive axially loaded cylindrical shells. International Journal of Solids and Structures, 115-116:73-86. https://doi.org/10.1016/j.ijsolstr.2017.02.028

Spagnoli, A. (2003). Koiter circles in the buckling of axially compressed conical shells. International Journal of Solids and Structures, 40(22):6095-6109. https://doi.org/10.1016/S0020-7683(03)00369-X

Wagner, H. N. R., Hühne, C., and Khakimova, R. (2019). On the development of shell buckling knockdown factors for imperfection sensitive conical shells under pure bending. Thin-Walled Structures, 145(August). https://doi.org/10.1016/j.tws.2019.106373 